\title{
Abelian surfaces with two plane cubic curve fibrations and Calabi-Yau threefolds
}

\author{
Klaus Hulek and Kristian Ranestad*
}

1. Introduction.

2. Numerical possibilities.

3. Elliptic scrolls and degenerate Calabi-Yau 3-folds in $\mathbf{P}^{5}$.

4. Non-normal Del Pezzo 3-folds in $\mathbf{P}^{5}$.

5. Non-normal Calabi-Yau 3-folds in $\mathbf{P}^{5}$.

6. Equations of elliptic scrolls in $\mathbf{P}^{5}$.

7. Heisenberg symmetry of elliptic scrolls in $\mathbf{P}^{5}$.

8. Conclusion.

\section{Introduction}

Abelian surfaces of small degree are contained in nodal Calabi-Yau 3-folds, similarly many Calabi-Yau 3-folds of small degree specialize to nodal Calabi-Yau 3folds with abelian surfaces on them. The first assertion is intimately connected with the fact that the moduli space of abelian surfaces of small degree is uniruled: An abelian surface on a Calabi-Yau 3-fold moves in a linear pencil, and therefore gives rise to a $\mathbf{P}^{1}$ in the moduli space of abelian surfaces. This idea was taken up and explored by Gross and Popescu [GP1, GP2] starting with a very singular Calabi-Yau variety, the secant variety of an elliptic normal curve. The translation scrolls inside the secant variety are degenerate abelian surfaces and form a $\mathbf{P}^{1}$ on the boundary of the moduli of abelian surfaces. They show that the secant variety deforms to nodal Calabi-Yau 3-folds with only isolated singularities and with a pencil of abelian surfaces as long as the degree of the elliptic curve is less than 11 . This limit is related to the Del Pezzo bound for the possible smoothing of minimal elliptic surface singularities.

* Both authors were partially supported by the HCM contract AGE (Algebraic Geometry in Europe), no ERBCHRXCT940557. The first author would also like to thank MSRI for its hospitality. 
We explore a similar setting. In a $\mathbf{P}^{2}$-scroll over an elliptic curve, any anticanonical divisor, if there is one, is a, possibly degenerate, abelian surface. If one can glue two $\mathbf{P}^{2}$-scrolls over elliptic curves along an anticanonical divisor, the union is a singular Calabi-Yau 3-fold. Furthermore, if the anticanonical divisor moves in a pencil on at least one of the two scrolls, then we are in a position like above.

We start in section 2 by asking for smooth abelian surfaces with two pencils of plane cubic curves on it. The two pencils would then define $\mathbf{P}^{2}$-scrolls whose union is Calabi-Yau. It turns out that purely numerical considerations bound the degree of these abelian surfaces by 18 . This bound is obtained by abelian surfaces which form the complete intersection $((0,3),(3,0))$ in the $\mathbf{P}^{2} \times \mathbf{P}^{2}$ with its Segre embedding. For each even degree $10 \leq d \leq 18$ there are numerical possibilities which are realized. In this paper we study the associated elliptic scrolls and CalabiYau 3 -folds in the case $d=12$, i.e. the case of abelian surfaces embedded linearly normally in $\mathbf{P}^{5}$.

In section 3 we find and describe the abelian surfaces of degree 12 and the two scrolls defined by their pencils of plane cubic curves. The union of the two scrolls is a non-normal Calabi-Yau 3-fold of degree 12.

In sections 4 and 5 a separate approach leads to constructions via projected Del Pezzo 3-folds of non-normal Calabi-Yau 3-folds in degrees 10,11, 12 and 13. The projected Del Pezzo 3-folds are bilinked to the non-normal Calabi-Yau 3-folds. In the last three sections we prepare the argument that the reducible Calabi-Yau 3 -folds described in section 3 may also be obtained via bilinkage from projected Del Pezzo 3-folds.

After finding equations for elliptic scrolls in section 6 , we devote section 7 to the Heisenberg symmetry of elliptic scrolls and provide a description of the family of Calabi-Yau 3-folds that are unions of two scrolls.

More precisely, let $H_{6}$ be the Heisenberg group of level 6 and let $N_{6}$ be its normalizer in $G L(6, \mathbf{C})$. In $N_{6}$ there is a natural involution $\iota$ which restricts to the abelian surfaces as multiplication by -1 . Let $G_{6}=\left\langle H_{6}, \iota\right\rangle$. Then the space of cubics in $\mathbf{P}^{5}$ contains a 4 -dimensional vector space of $G_{6}$-invariant pencils.

We let $\mathbf{P}=\mathbf{P}^{3}$ be the parameter space for these $G_{6}$-invariant pencils of cubics. $H_{6}$ contains a subgroup isomorphic to $H_{2}$ and four subgroups $H\left(K_{1}\right), \ldots, H\left(K_{4}\right)$ of index 3 containing this subgroup. For every subgroup $H\left(K_{i}\right)$ there is a set of three lines in $\mathbf{P}^{5}$ containing $H\left(K_{i}\right)$ in its stabilizer and left invariant by the action of $H_{6}$. Similarly there exists for each subgroup $H\left(K_{i}\right)$ a line $l_{i}$ in $\mathbf{P}$ parametrizing pencils of cubics which contain the corresponding three lines.

A general point on any of these four lines $l_{i}$ in $\mathbf{P}$ corresponds to a pencil of cubics which defines an elliptic scroll singular along the corresponding three lines in $\mathbf{P}^{5}$. The scroll is residual to three $\mathbf{P}^{3}$ 's in the complete intersection of the two cubics.

Between each pair of lines $l_{i}, l_{j}$, there is a $1: 1$ correspondence defined by the pairs of points which correspond to elliptic scrolls that intersect along a possibly degenerate abelian surface. The lines spanned by corresponding points form a conic section in the Grassmannian of lines in $\mathbf{P}$. Altogether there are 6 disjoint conic 
sections in the Grassmannian of lines in $\mathbf{P}$ which parametrize abelian surfaces with two plane cubic curve fibrations.

In the final section we state and prove the main theorem of the paper: Let $X_{E}$ and $X_{F}$ be elliptic scrolls in $\mathbf{P}^{5}$ that intersect precisely along a $(1,6)$-polarized abelian surface.

Theorem 8.3. The reducible 3 -fold $Y=X_{E} \cup X_{F}$ is a degeneration of irreducible non-normal Calabi-Yau 3-folds of degree 12 in $\mathbf{P}^{5}$. The general such 3 -fold is singular precisely along 6 disjoint lines.

Chang has described smooth 3 -folds of degree 12 that are birational to CalabiYau 3-folds (cf. [Ch], [DP]). Similar to the ones described in this paper they are bilinked to Fano 3-folds of degree 7, but they differ by their sectional genus. This difference is manifested in the appearance of non-normal singularities. The CalabiYau 3-folds of Theorem 8.3 do not deform to smooth ones: The double point formulas for 3 -folds in $\mathbf{P}^{5}$ give the class of the non-normal singular locus in terms of the coefficients of the Hilbert polynomial, so any deformation also has nonnormal singularities.

We find three open problems related to the topics of this paper particularly interesting:

Problem 1.1. Find $H_{6}$-invariant non-normal but irreducible Calabi-Yau 3-folds, with a pencil of $(1,6)$-polarized abelian surfaces, degenerating to the union of two elliptic scrolls.

Problem 1.2. Consider the normalization of the Calabi-Yau 3-folds of degrees $10, \ldots, 13$ of section 5. Find the invariants, the Betti- and Hodge numbers and and describe the Kähler cone of these Calabi-Yau 3-folds.

Problem 1.3. Describe the elliptic scrolls and the reducible Calabi-Yau 3-folds in the cases $d=14,16,18$.

We work over the complex numbers.

\section{Numerical possibilities}

Assume that an abelian surface $A \subset \mathbf{P}^{n}$ has two fibrations

$$
p: A \rightarrow E \quad \text { and } \quad q: A \rightarrow F
$$

in plane cubic curves. These fibrations define two $\mathbf{P}^{2}$-scrolls. If the fibers of the maps $p$ and $q$ have intersection number $\geq 2$, then the planes in the two fibrations intersect in at least a line and the two $\mathbf{P}^{2}$-scrolls coincide. So for our purposes we can assume that $F$ and $E$ are sections of $p$ and $q$ with $E \cdot F=1$. In particular $A=E \times F$. 
When there is no isogeny between $E$ and $F$ then $A$ is the product of $E \times F$ in the Segre embedding of $\mathbf{P}^{2} \times \mathbf{P}^{2}$ in $\mathbf{P}^{8}$. We shall now assume that $E$ is general in the sense that $E$ has $\operatorname{End} E \cong \mathbf{Z}$ and that $\gamma: E \rightarrow F$ is a primitive isogeny of degree $l$. Then $N S(A)$ is generated by $E, F$ and $\Gamma$ where $\Gamma$ is the graph of $\gamma$. The numerical equivalence of a hyperplane divisor on $A$ may therefore be expressed as

$$
H \equiv a E+b F+c \Gamma, \quad a, b, c \subset \mathbf{Z} .
$$

Notice that these surfaces and these divisors really exist for any general elliptic curve $E$. We investigate for which numerical data they give us two plane cubic curve fibrations. The intersection numbers are given by the table:

$\begin{array}{cccc} & E & F & \Gamma \\ E & 0 & 1 & l \\ F & 1 & 0 & 1 \\ \Gamma & l & 1 & 0 .\end{array}$

With the requirements

$$
H \cdot E=b+l c=3, \quad H \cdot F=a+c=3 \quad \text { and } \quad H \cdot \Gamma=a l+b \geq 2,
$$

we get

$$
d / 2:=H^{2} / 2=9-l c^{2} .
$$

This means that $d / 2 \leq 9$ and that there are the following possibilities for $H$ :

Table 1.

\begin{tabular}{llllcc}
\hline$d$ & $l$ & $c$ & $a$ & $b$ & $H \cdot \Gamma$ \\
\hline 10 & 1 & 2 & 1 & 1 & 2 \\
10 & 4 & 1 & 2 & -1 & 7 \\
12 & 3 & 1 & 2 & 0 & 6 \\
14 & 2 & 1 & 2 & 1 & 5 \\
16 & 1 & 1 & 2 & 2 & 4 \\
18 & $*$ & 0 & 3 & 3 & $*$ \\
\hline
\end{tabular}

The $*$ in table 1 means that there is no isogeny between $E$ and $F$ involved.

Proposition 2.1. There exist abelian surfaces with two plane curve fibrations of degree $d$ in $\mathbf{P}^{\frac{d}{2}-1}$, when $d=10,12,14,16,18$.

Proof. To give examples it is now enough to check that $H$ is very ample. For this we use Reider's criterion [Re], which in these cases reduces to check that there are no elliptic curves $C$ on $A$ with $H \cdot C \leq 2$. Any such curve $C$, not equivalent to $E$, $F$ or $\Gamma$, must intersect each of these strictly positively, i.e.

$$
C \cdot E>0, \quad C \cdot F>0, \quad \text { and } C \cdot \Gamma>0 .
$$

But in each case except the second $H=a E+b F+c \Gamma$ with $a+b+c \geq 3$ so $H \cdot C \geq 3$. In the second case $H=2 E-F+\Gamma$, so if $C=\alpha E+\beta F+\gamma \Gamma$, then 
$H \cdot C<3$ implies that

$$
3 \alpha+3 \beta+7 \gamma \leq 2 .
$$

The other inequalities above yield

$$
\beta+4 \gamma \geq 1, \quad \alpha+\gamma \geq 1 \text { and } 4 \alpha+\beta \geq 1,
$$

while $C^{2}=0$, since $C$ is elliptic, yields

$$
\alpha \beta+4 \alpha \gamma+\beta \gamma=0 .
$$

From the first four inequalities we get $\beta \leq-(\alpha+\gamma)$ which combines with the last equality to yield

$$
0=4 \alpha \gamma+\beta(\alpha+\gamma) \leq 4 \alpha \gamma-(\alpha+\gamma)^{2}=-(\alpha-\gamma)^{2} .
$$

This only occurs when $\alpha=\gamma$, i.e. from the relation $C^{2}=0$, when $\alpha=0$ or $2 \alpha+\beta=0$. The former is impossible since $\alpha+\gamma>0$, while the latter is impossible since then $C \cdot H=10 \alpha-6 \alpha=4 \alpha \geq 4$.

In the first case $H \cdot \Gamma=2$, so $H$ is not very ample. In fact $|H|$ maps $A$ two to one to a quintic elliptic scroll in $\mathbf{P}^{4}$. In this case $E=F$ and the scroll is the symmetric product of $E$. Thus the two fibrations coincide in the image. In each of the other cases $|H|$ defines an embedding.

Remark 2.2. When $d=18$ there is the simple example of

$$
E \times F=E \times \mathbf{P}^{2} \cap \mathbf{P}^{2} \times F \subset \mathbf{P}^{2} \times \mathbf{P}^{2}
$$

in its Segre embedding in $\mathbf{P}^{8}$. Clearly the union of the two scrolls deform in this case to Calabi-Yau 3-folds.

In this paper we shall concentrate on the case $d=12$.

\section{Elliptic scrolls and degenerate Calabi-Yau 3-folds in $\mathrm{P}^{5}$}

From now on we consider abelian surfaces $A \subset \mathbf{P}^{5}$ of degree 12 , i.e. with a $(1,6)$ polarization, and with two fibrations

$$
p: A \rightarrow E \quad \text { and } \quad q: A \rightarrow F
$$

in plane cubic curves. Furthermore we assume that $E$ is general. We denote by $X_{E}$ the scroll of planes of the fibration $p$, and by $X_{F}$ the scroll of planes of the fibration $q$. The corresponding $\mathbf{P}^{2}$-bundles are denoted $V_{E}$ and $V_{F}$, respectively.

In the notation of the previous section and table 1 there is an isogeny

$$
\gamma: E \rightarrow F
$$


of degree 3. Furthermore, if $\Gamma$ is the graph of the isogeny, then the hyperplane divisor is

$$
H=2 E+\Gamma .
$$

We fix an origin $o \in E$ and let $s_{1}=\gamma\left(t_{1}\right)$, for some 3-torsion point $t_{1}$ on $E$, not in the kernel of $\gamma$. Let $h: F \rightarrow F$ be translation by $s_{1}$, and let $\gamma^{*}: \operatorname{Pic}^{0} F \rightarrow \operatorname{Pic}^{0} E$ be the isogeny dual to $\gamma$. If $\mathcal{O}_{F}(o)$ is the line bundle of degree 1 whose unique section vanishes at $o$, then $h^{*}\left(\mathcal{O}_{F}(o)\right) \otimes \mathcal{O}_{F}(-o)$ generates the kernel of $\gamma^{*}$, i.e.

$$
\text { ker } \gamma^{*}=\left\{\mathcal{O}_{F}, h^{*}\left(\mathcal{O}_{F}(o)\right) \otimes \mathcal{O}_{F}(-o),\left(h^{2}\right)^{*}\left(\mathcal{O}_{F}(o)\right) \otimes \mathcal{O}_{F}(-o)\right\} \quad\left(h^{3}=\mathrm{id}\right) \text {. }
$$

Proposition 3.1. The rank 3 vector bundle associated to the $\mathbf{P}^{2}$-bundle $V_{F}$ decomposes into $\mathcal{E}=\mathcal{L}_{0} \oplus h^{*} \mathcal{L}_{0} \oplus\left(h^{2}\right)^{*} \mathcal{L}_{0}$, where $\mathcal{L}_{0}$ is a line bundle of degree 2 on $F$ and $h$ is as above. Furthermore the scroll $X_{F}$ is singular precisely along three lines, which span $\mathbf{P}^{5}$.

Proof. First, notice that $\Gamma$ is contained in a pencil of hyperplanes defined by the pencil $|H-\Gamma|=|2 E|$ on $A$. This pencil is the pullback by $q$ of a divisor $\Delta_{0}$ of degree 2 on $F$. Since any divisor in this pencil is a pair of plane cubic curves contained in a hyperplane, their planes intersect in a point. Thus the linear system $\left|\Delta_{0}\right|$ defines a morphism of $F$ of degree 2 into the double locus of $X_{F}$.

Next we consider the translates $\Gamma_{t}$ of $\Gamma$ on $A$ by a point on $t$ on $E$ and find which $\Gamma_{t}$ are contained in a pencil of hyperplanes. This happens precisely when $H-\Gamma_{t}$ is the pullback of a divisor of degree 2 from $F$, or equivalently when the restriction of $\Gamma-\Gamma_{t}$ to a fiber $E_{f}=q^{-1}(f)$ of $q$ is trivial. But the translate $\Gamma_{t}$ can be represented as

$$
\{(x+t, \gamma(x)) \mid x, t \in E\}
$$

so the intersection

$$
\left(\Gamma-\Gamma_{t}\right) \cap E_{f}=\gamma^{-1}(f)-\gamma^{-1}(f)+3 t
$$

which is trivial precisely when $3 t=o$. Since $\gamma$ is already an isogeny of degree 3 , the 3 -torsion points in the kernel of $\gamma$ leave $\Gamma$ invariant under translation, so in fact we may choose $t=t_{1}$ as above and we get 3 distinct translates of $\Gamma$ which are contained in a pencil of hyperplanes in $\mathbf{P}^{5}$. We denote them by $\Gamma_{0}(=\Gamma), \Gamma_{1}, \Gamma_{2}$. They each span a $\mathbf{P}^{3}$ and determine a linear system of degree 2 on $F$, which we denote by $\left|\Delta_{0}\right|,\left|\Delta_{1}\right|,\left|\Delta_{2}\right|$. Each linear system $\left|\Delta_{i}\right|$ defines a morphism of degree 2 of $F$ to the double locus of $X_{F}$. The images of these three maps are disjoint lines, and clearly the planes of $X_{F}$ are spanned by the respective images by these three maps. Therefore $V_{F}$ is defined by a decomposable rank 3 bundle $\mathcal{E}$ of degree 6 . If we denote the line bundle associated to the divisor $\Delta_{i}$ by $\mathcal{L}_{i}$, then

$$
\mathcal{E}=\mathcal{L}_{0} \oplus \mathcal{L}_{1} \oplus \mathcal{L}_{2}
$$

To check the differences between the line bundles $\mathcal{L}_{i}$, we consider the intersection

$$
\left(\Gamma-\Gamma_{t}\right) \cap F_{e}=\gamma(e)-\gamma(e-t)=\gamma(t) .
$$


Since $3 t_{1}=o$, translation by $s_{1}=\gamma\left(t_{1}\right)$ on $F$ is a 3 -torsion element that generates the kernel of the dual isogeny, $\gamma^{*}: \operatorname{Pic}^{0} F \rightarrow \operatorname{Pic}^{0} E$, i.e.

$$
\mathcal{L}_{i}=\left(h^{i}\right)^{*} \mathcal{L}_{0}
$$

where $h$ is the translation on $F$ by $s_{1}$.

It remains only to check the singularities of $X_{F}$. Any singular point of $X_{F}$ is the intersection of two, possibly infinitely close, planes of $X_{F}$. But two planes intersect only if they span at most a hyperplane. The two planes are defined by a section of a line bundle $q^{*} \mathcal{L}$ for a line bundle $\mathcal{L}$ of degree 2 on $F$. They intersect precisely when

$$
\mathcal{E} \otimes \mathcal{L}^{-1}
$$

has a section. But this is the case precisely when $\mathcal{L}=\mathcal{L}_{i}$ for $i \in\{0,1,2\}$. Furthermore, for each of these three cases $\mathcal{E} \otimes \mathcal{L}_{i}^{-1}$ has precisely one section, so the corresponding planes span a hyperplane and the intersection of the two planes is only a point.

The abelian surface $A$ is an anticanonical divisor on $V_{F}$. We compute the sections of $-K_{V_{F}}$.

Lemma 3.2. $\mathrm{h}^{0}\left(\mathcal{O}_{V_{F}}\left(-K_{V_{F}}\right)\right)=4$.

Proof. The natural isomorphism

$$
\mathrm{H}^{0}\left(\mathcal{O}_{V_{F}}\left(-K_{V_{F}}\right)\right) \cong \mathrm{H}^{0}\left(F, \operatorname{Sym}^{3} \mathcal{E} \otimes \mathcal{L}_{0}^{-1} \otimes \mathcal{L}_{1}^{-1} \otimes \mathcal{L}_{2}^{-1}\right)
$$

reduces the computation to counting trivial summands of the rank 10 vector bundle

$$
\operatorname{Sym}^{3} \mathcal{E} \otimes \mathcal{L}_{0}^{-1} \otimes \mathcal{L}_{1}^{-1} \otimes \mathcal{L}_{2}^{-1} .
$$

Since $\mathcal{L}_{0}^{-1} \otimes \mathcal{L}_{1}^{-1} \otimes \mathcal{L}_{2}^{-1}=\mathcal{L}_{0}^{-3}$ this count is the number of summands in

$$
\operatorname{Sym}^{3} \mathcal{E}=\operatorname{Sym}^{3}\left(\mathcal{L}_{0} \oplus h^{*} \mathcal{L}_{0} \oplus\left(h^{2}\right)^{*} \mathcal{L}_{0}\right)
$$

which equal $\mathcal{L}_{0}^{3}$. As $h^{3}=$ id this number is 4 .

Lemma 3.3. The $\mathbf{P}^{2}$-bundle $V_{F}$ is the quotient of a trivial bundle $\mathbf{P}^{2} \times E$ by a cyclic group of order 3 .

Proof. Consider the isogeny $\gamma: E \rightarrow F$ again. Since $h^{*}\left(\mathcal{O}_{F}(o)\right) \otimes \mathcal{O}_{F}(-o)$ generates the kernel of $\gamma^{*}$ the isogeny dual to $\gamma$,

$$
\gamma^{*} \mathcal{L}_{0} \cong \gamma^{*} h^{*} \mathcal{L}_{0} \cong \gamma^{*}\left(h^{2}\right)^{*} \mathcal{L}_{0}
$$

Therefore the pullback of $V_{F}=\mathbf{P}(\mathcal{E})$ over $F$ via $\gamma$ trivializes the bundle. The kernel of $\gamma$ is a cyclic group of order three which acts on the pullback $\gamma^{*} \mathcal{E}$.

Thus we may construct $V_{F}$ by starting with $\mathbf{P}^{2} \times E$, and dividing by a suitable diagonal action of the cyclic group of order 3 . For this we consider a vector space 
$V=\left\langle x_{0}, x_{1}, x_{2}\right\rangle$ with the action

$$
\tau: x_{i} \mapsto \epsilon^{i} x_{i}, \quad i \in \mathbf{Z}_{3} .
$$

As above, let $o \in E$ be the origin of $E$, and consider the linear system $|3 o|$ on $E$. It embeds $E$ as a plane cubic curve in $\mathbf{P}^{2}$. We may choose $\left\langle e_{0}, e_{1}, e_{2}\right\rangle$ as a basis for the underlying vector space of $\mathbf{P}^{2}$ such that

$$
\tau: e_{i} \mapsto \epsilon^{-i} e_{i}
$$

induces the action of translation by a 3 -torsion point $t_{0}$ on $E$. The diagonal action defined by $\tau \in \mathbf{Z}_{3}$ on $V \times E$ :

$$
\tau: v \times e \mapsto \tau(v) \times e+t_{0}
$$

acts without fixed points, so the quotient is a rank 3 vector bundle on $E /\left\langle t_{0}\right\rangle=F$. The action of $\tau$ on $V$ decomposes into the characters

$$
V=\left\langle x_{0}\right\rangle \oplus\left\langle x_{1}\right\rangle \oplus\left\langle x_{2}\right\rangle .
$$

The anticanonical divisors on $V_{F}$ pull back to anticanonical divisors on $\mathbf{P}^{2} \times E$ which are invariant under the action of $\tau$. But the anticanonical divisors on $\mathbf{P}^{2} \times E$ are just the pullbacks of the cubic curves on the plane. The action of $\tau$ on the plane has the following basis of invariant cubics:

$$
\left\langle x_{0}^{3}, x_{1}^{3}, x_{2}^{3}, x_{0} x_{1} x_{2}\right\rangle .
$$

Since these have no basepoints, there are no basepoints for the system of anticanonical divisors on $V_{F}$, and the general one is smooth. Notice furthermore that this linear system of invariant cubics contains the Hesse pencil

$$
\left\langle x_{0}^{3}+x_{1}^{3}+x_{2}^{3}, x_{0} x_{1} x_{2}\right\rangle,
$$

and recall that the singular curves in this pencil are 4 triangles. In fact it is easy to check that these four triangles are the only triangles in the linear system of invariant cubics. The vertices of the triangle $x_{0} x_{1} x_{2}=0$ are mapped to the singular lines of $X_{F}$. The vertices of the three other triangles sweep out elliptic normal curves of degree 6 as we shall see next.

Proposition 3.4. The scroll $X_{F}$ is the 3-torsion translation scroll of an elliptic normal curve in $\mathbf{P}^{5}$.

Proof. Consider an elliptic normal curve $C$ of degree 6 in $\mathbf{P}^{5}$, embedded by the linear system $|6 o|$. For any $P \in C$ consider the translation scroll

$$
V_{P}=\cup_{y \in C}\langle y, y+P, y+2 P\rangle .
$$

This is, for general $P$, a $\mathbf{P}^{2}$-scroll of degree 18 . When $3 P=o$ the points $y, y+P$ and $y+2 P$ generate the same plane, so then the translation scroll has degree 6 . In this case

$$
\langle y, y+P, y+2 P\rangle \cap\langle z, z+P, z+2 P\rangle \neq \emptyset
$$


precisely when $3 y+3 z=o$. But then the pencil $|y+z|$ defines a map from $C$ to the double locus of $V_{P}$. Now $3(y+z)=3(y+z+P)=3(y+z+2 P)$, so this map factors through the isogeny $C \rightarrow C /\langle P\rangle$. Thus the 9 linear systems $|y+z|$ with $3(y+z)=0$ define three pencils of pairs of planes each defining a double line for the translation scroll. The translation scroll is clearly a scroll over $C /\langle P\rangle$ and its associated vector bundle decomposes into the sum of three line bundles of degree 2 which define the three double lines. The differences between any two of these line bundles are the powers of some 3-torsion line bundle. If $C \cong E$ and $C /\langle P\rangle=F$, we have $V_{P} \cong X_{F}$.

Finally, we consider the union of the two elliptic scrolls $X_{1}=X_{E}$ and $X_{2}=X_{F}$ in $\mathbf{P}^{5}$ which both contain the $(1,6)$-polarized abelian surface $A$. Let $V_{1}=V_{E}$ and $V_{2}=V_{F}$ be the corresponding $\mathbf{P}^{2}$-bundles. Then $V_{1}$ and $V_{2}$ are the normalizations of $X_{1}$ and $X_{2}$ and $A \in\left|-K_{V_{1}}\right|$, resp. $A \in\left|-K_{V_{2}}\right|$. Let $Y=X_{1} \cup X_{2}$.

Proposition 3.5. $Y$ has a partial desingularization $Y_{0}=V_{1} \cup V_{2}$, which is a Calabi-Yau 3-fold, i.e. $K_{Y_{0}}=\mathcal{O}_{Y_{0}}$ and $q=\mathrm{h}^{1}\left(\mathcal{O}_{Y_{0}}\right)=0$. In particular $V_{1} \cap V_{2}=A$.

Proof. First, we may use the previous notation and let $A=E \times F$ with hyperplane divisor $H=2 E+\Gamma$.

Notice that the abelian surface $A$ does not intersect the singular lines of $X_{F}$. In fact $\left|H-E_{f}\right|$, for a plane cubic curve $E_{f}$ passing through a singular point, would then have a basepoint. This is impossible by Reider's criterion [Re].

We show next that $X_{1}$ and $X_{2}$ intersect transversally along $A$ : Near $A$ the 3 -fold $Y$ is the intersection of two smooth 3-folds. If the intersection is not transversal the tangent spaces of the two scrolls at some point coincide. This is possible only if the planes of the two scrolls at the given point intersect along a line. Those two planes intersect $A$ in elliptic cubic curves $E_{f}$ and $F_{e}$ which meet in a point. Any curve in $\left|H-E_{f}-F_{e}\right|$ has degree 6 and arithmetic genus 2. Since there is a pencil of hyperplanes through the two planes, this curve moves in a pencil on $A$, this is impossible by Riemann Roch, so transversality follows. If $X_{1} \cap X_{2}$ contains a point disjoint form $A$, then a plane in $X_{1}$ meets a plane in $X_{2}$ along a line and the argument above applies again. Thus $X_{1}$ and $X_{2}$ meet transversally along $A$.

Now, look at the partial desingularization $Y_{0}$ of $Y$ obtained by normalizing $X_{1}$ and $X_{2}$. Thus we may write $Y_{0}=V_{1} \cup V_{2}$ and $V_{1} \cap V_{2}=A$. To show that $q\left(Y_{0}\right)=0$ we consider $\operatorname{Pic}^{0} Y_{0}$. An element in $\operatorname{Pic}^{0} Y_{0}$ is a pair $\left(\mathcal{M}_{1}, \mathcal{M}_{2}\right)$ with $\mathcal{M}_{i} \in \operatorname{Pic}^{0} V_{i}$ which glue along $A$. Consider the fibrations

$$
p_{1}: V_{1} \rightarrow F \quad \text { and } \quad p_{2}: V_{2} \rightarrow E .
$$

Now, $\mathcal{M}_{i}=p_{i}^{*}\left(\mathcal{N}_{i}\right)$, where $\mathcal{N}_{1} \in \operatorname{Pic}^{0}(F)$ resp. $\mathcal{N}_{2} \in \operatorname{Pic}^{0}(E)$. On $A$ we have sections of each $p_{i}$ which are fibres of the opposite map. We identify these sections with $F$ and $E$ respectively. Then $\left.\mathcal{M}_{1}\right|_{E}=\mathcal{O}_{E}$ and $\left.\mathcal{M}_{2}\right|_{F}=\mathcal{O}_{F}$. So if we want to glue $\mathcal{M}_{1}$ and $\mathcal{M}_{2}$ we must have that

$$
\mathcal{O}_{E}=\left.\mathcal{M}_{1}\right|_{E}=\mathcal{N}_{2}
$$


and

$$
\mathcal{O}_{F}=\left.\mathcal{M}_{2}\right|_{F}=\mathcal{N}_{1}
$$

and hence $\left(\mathcal{M}_{1}, \mathcal{M}_{2}\right)=\left(\mathcal{O}_{X_{1}}, \mathcal{O}_{X_{2}}\right)$. This shows that $\operatorname{Pic}^{0} Y_{0}=\left\{\mathcal{O}_{Y_{0}}\right\}$. Since $\operatorname{Pic}^{0} Y_{0}$ is a reduced group scheme in characteristic 0 and since $\mathrm{H}^{1}\left(X, \mathcal{O}_{Y_{0}}\right)$ is the tangent space at the origin, it follows that $q\left(Y_{0}\right)=\mathrm{h}^{1}\left(Y_{0}, \mathcal{O}_{Y_{0}}\right)=0$.

Since $A \in\left|-K_{V_{1}}\right|$, resp. $A \in\left|-K_{V_{2}}\right|$ it is clear that the restriction of $K_{Y_{0}}$ to $V_{1}$ and to $V_{2}$ is trivial. But $\operatorname{Pic}^{0} Y_{0}=\left\{\mathcal{O}_{Y_{0}}\right\}$, so $K_{Y_{0}}=\mathcal{O}_{Y_{0}}$.

The 3-fold $Y=X_{E} \cup X_{F}$ is non-normal, singular along six lines three on each scroll in addition to the surface $A=X_{E} \cap X_{F}$. In the next section we describe a series of non-normal Del Pezzo 3-folds. In section 5 we show that these are bilinked to non-normal Calabi-Yau 3-folds. After some further analysis of the equations of $Y$ in sections 6 and 7 we show in section 8 that $Y$ is a degeneration of these non-normal Calabi-Yau 3-folds.

\section{Non-normal Del Pezzo 3-folds in $\mathrm{P}^{5}$}

Del Pezzo 3 -folds are 3 -folds $W$ for which $K_{W} \cong-2 H$, where $H$ is ample. Accordingly any smooth surface on $W$ in $|H|$ is a Del Pezzo surface.

Let $V_{t} \subset \mathbf{P}^{t+1}$ with $t=3, \ldots, 8$ be the image of $\mathbf{P}^{3}$ by the map defined by all quadrics through $8-t$ general points in $\mathbf{P}^{3}$. Then $V_{t}$ is a Del-Pezzo 3 -fold. We describe the image $W_{t} \subset \mathbf{P}^{5}$ of the general projection of $V_{t}$.

In particular we want to describe the singular non-normal locus. Thus we are interested in the cases $t=5,6,7,8$ and will prove

Theorem 4.1. $W_{t}$ is non-normal along $\left(\begin{array}{c}t-3 \\ 2\end{array}\right)$ skew lines and has $\left(\begin{array}{c}8-t \\ 2\end{array}\right)$ additional ordinary double points when $t=5,6,7,8$.

To prove this we will use a result of Reye on linear systems of quadrics. To explain Reye's result we need the notion of apolarity applied to quadrics in $\mathbf{P}^{3}$. Thus let $S=k\left[x_{0}, \ldots, x_{3}\right]$ and $T=k\left[y_{0}, \ldots, y_{3}\right]$, and define a pairing $S_{2} \times T_{2} \rightarrow k$ by letting $S$ operate as differential operators on $T$ and vica versa.

We say that quadrics in $S$ and $T$ are apolar if they are orthogonal with respect to this pairing. In our situation we think of $S$ as the coordinate $\operatorname{ring}$ of $\mathbf{P}^{3}$ and $T$ as the coordinate ring of $\check{\mathbf{P}}^{3}$. Starting with a 6 -dimensional subspace of quadrics $V \subset S_{2}$, there is a 4-dimensional subspace i.e. a web of quadrics $V^{\perp} \subset T_{2}$.

Since any quadric in $V$ is apolar to any member of $V^{\perp}$, we say that $V$ and $V^{\perp}$ are apolar sets of quadrics. Consider the discriminant $D$ of the space $V^{\perp}$ of quadrics. This is a quartic surface defined by the determinant of a symmetric $4 \times 4$ matrix with linear entries. The quadrics in $V^{\perp}$ of rank 1 and 2 are respectively triple and double points on $D$. The possible numbers of rank 1 and rank 2 quadrics are given in the following table: 
Table 2.

\begin{tabular}{cc}
\hline rank 1 quadrics & rank 2 quadrics \\
\hline 4 & $0 / \infty$ \\
3 & 1 \\
2 & 3 \\
1 & 6 \\
0 & 10 \\
\hline
\end{tabular}

This follows from a few lemmas which have independent interest for us.

Lemma 4.2. Each rank 1 quadric in $V^{\perp}$ determines a basepoint for the quadrics in $V$ and vice versa. Each rank 2 quadric in $V^{\perp}$ determines a line contained in 4 quadrics in $V$ and vica versa. Alternatively, if $\rho$ is the map defined by the linear system $V$ of quadrics, then each rank 2 quadric in $V^{\perp}$ determines a line in the source double point locus of $\rho$ and vice versa.

Proof. Note that if $a=\left(a_{0}, a_{1}, a_{2}, a_{3}\right), L=\sum_{i=0}^{3} a_{i} y_{i}$ and $q \in S_{2}$, then

$$
L^{2}(q)=2 q(a) .
$$

Now each rank 1 quadric in $V^{\perp}$ has the form $L^{2}$ for some point $a$, and apolarity says that $L^{2}(q)=2 q(a)=0$ for every quadric $q$ in $V$ so $a$ is a basepoint for $V$. Conversely, if $a=\left(a_{0}, a_{1}, a_{2}, a_{3}\right)$ is a basepoint for $V$ and $L=\sum_{i=0}^{3} a_{i} y_{i}$, then $L^{2}(q)=2 q(a)=0$ for every $q \in V$ so $L^{2} \in V^{\perp}$.

Each rank 2 quadric in $V^{\perp}$ has the form $L_{1}^{2}+L_{2}^{2}$ for some linear forms $L_{1}=$ $\sum_{i=0}^{3} a_{i} y_{i}$ and $L_{2}=\sum_{i=0}^{3} b_{i} y_{i}$. Let $l \subset \mathbf{P}^{3}$ be the line spanned by the points $a=\left(a_{0}, a_{1}, a_{2}, a_{3}\right)$ and $b=\left(b_{0}, b_{1}, b_{2}, b_{3}\right)$. Let $V_{l} \subset V$ be the subspace of quadrics which vanish on $l$. Then

$$
V_{l}=\left\{q \in V \mid L_{1}^{2}(q)=2 q(a)=L_{2}^{2}(q)=2 q(b)=L_{1} L_{2}(q)=0\right\} .
$$

But $\left(L_{1}^{2}+L_{2}^{2}\right)(q)=0$ for every $q \in V$, so $V_{l}$ has codimension 2 in $V$. Conversely if $V_{l}$ has codimension 2 in $V$, then some linear combination of $L_{1}^{2}, L_{2}^{2}$ and $L_{1} L_{2}$ is contained in $V^{\perp}$. But any such linear combination is a rank 2 (or rank 1) quadric, and the lemma follows.

Porteous' formula [cf. Fu 14.4.11] computes the number of rank 2 quadrics in a general web of rank 4 quadrics. This number is 10 . Reye found a geometric interpretation of these 10 rank 2 quadrics considering apolar twisted cubic curves to the web of quadrics, i.e. twisted cubic curves whose defining net of quadrics is apolar to $V^{\perp}$.

By a determinental net of quadrics we mean a net (i.e. a 3-dimensional space) of quadrics which is generated by the $2 \times 2$ minors of a $2 \times 3$ matrix of linear forms. The general determinental net of quadrics generates the ideal of a twisted cubic curve. 
Lemma 4.3. (Reye). The general 6-dimensional subspace $V \subset S_{2}$ contains precisely two determinental nets of quadrics, which together span $V$. If $C_{1}$ and $C_{2}$ are the twisted cubic curves defined by these two nets, then every rank 1 quadric in $V^{\perp}$ corresponds to a point of intersection between $C_{1}$ and $C_{2}$ and vice versa. Furthermore every rank 2 quadric in $V^{\perp}$ corresponds to a common secant line for $C_{1}$ and $C_{2}$ and vice versa.

Proof. The number of determinental nets of quadrics in a general 6-dimensional space $V$ of quadrics is nowadays computable by quantum cohomology [Kre] (compute the number of twisted cubic surface scrolls through nine points in $\mathbf{P}^{4}$ in the quantum cohomology of the Grassmannian of lines and intersect with a general $\mathbf{P}^{3}$ ), a few years ago by modern intersection theory [ES] and in the ancient times by direct geometric arguments [Rey]. We leave the choice of reference to the reader.

Given two determinental nets which span $V$, the correspondence between points of intersection and the rank 1 quadrics follows from Lemma 4.2.

For the second correspondence consider first a common secant line to $C_{1}$ and $C_{2}$. This line and any one of the two curves form a complete intersection $(2,2)$. Therefore the line lies in a pencil of quadrics from each of the two determinental nets. Together the two pencils form a web of quadrics in $V$ which by Lemma 4.2 corresponds to a rank 2 quadric in $V^{\perp}$. On the other hand the secant lines to a twisted cubic curve form a congruence of bidegree $(1,3)$ in the Grassmannian of lines in $\mathbf{P}^{3}$. Thus two general twisted cubic curves have

$$
(1,3) \cdot(1,3)=1+9=10
$$

common secant lines. This is exactly the number of rank 2 quadrics in the web $V^{\perp}$, so the second correspondence follows.

To fill in the remainder of table 2 we want to compute how much the number of rank 2 quadrics decreases when the web acquires a rank 1 quadric. We give an argument using Reye's geometric interpretation. Our arguments will depend on a genericity assumption, i.e. the space $V$ is general with a given number of basepoints. The argument would go through without this assumption also, but then the numbers in table 2 would have different interpretations. Since we will only use general systems $V$ we do not consider the degenerate cases.

When the space $V$ of quadrics have basepoints, then there will be infinitely many apolar twisted cubic curves to $V$, but taking two of them will always suffice for our argument. First note that as long as the two twisted cubic curves have less than 4 common points, the corresponding nets of quadrics do not intersect.

When the web contains one rank 1 quadric, then the two twisted cubic curves have one common point. The number of common secant lines passing through this point is, with our genericity assumption, easily computed by projection from the point, it is 4 , the number of intersection points between two conics in the plane. So the web has 6 rank 2 quadrics in addition to the rank 1 quadric. This is the second row in the table. 
When the web contains 2 rank 1 quadrics, then the two twisted cubic curves have two common points. In this case there are 4 common secant lines through each of the two intersection points, and one of these is the line passing through the two points, so there are exactly 3 common secant lines which do not pass through any of the two common points. Thus the web has 3 rank 2 quadrics in addition to the two rank 1 quadrics. This is the third row in the table.

When the web contains 3 rank 1 quadrics, the two twisted cubic curves have 3 points in common. There are 4 common secant lines through each intersection point, and adding up three are counted twice, so we get only one common secant line which does not pass through any of the intersection points. Thus the web has one rank 2 quadric in addition to the three rank 1 quadrics. This is the fourth row in the table.

When the web has more than 3 rank 1 quadrics, the twisted cubics have at least 4 points of intersection. In this case the number of common secant lines that does not pass through the intersection points is infinite or zero depending on whether the two determinental nets intersect or not. This covers the remaining row in the table.

Lemma 4.4. Consider the map $\rho$ defined by the linear system $V$ of quadrics, and consider a subscheme $Z$ of length 2 which does not intersect the baselocus of $V$. Then $Z$ is mapped to a point by $\rho$ if and only if either the restriction of $\rho$ to the unique line passing through $Z$ is $2: 1$ onto a line, or this line contains two base points for $V$.

Proof. The linear system $V$ restricted to the line through $Z$ has degree 2 . If this linear system has no basepoints, then the image of the line by $\rho$ is a line or a conic section. It is a line if and only if some subscheme of length 2 is mapped to a point. If the line through $Z$ intersects the baselocus, the intersection must contain two points, such that the line is contracted by $V$.

Proof of Theorem 4.1. Combining Lemmas 4.2, 4.3 and 4.4 we find that a general 6 -dimensional linear system of quadrics with $8-t$ basepoints and $t=5,6,7,8$ defines a rational map from $\mathbf{P}^{3}$ to $\mathbf{P}^{5}$, whose image has degree $t$ and whose nonnormal double locus consists of $\left(\begin{array}{c}t-3 \\ 2\end{array}\right)$ disjoint lines. Any line between basepoints is contracted to an isolated singularity. The number of isolated singular points is therefore $\left(\begin{array}{c}8-t \\ 2\end{array}\right)$. It is easily checked by restriction to general hyperplane sections that these singular points are ordinary double points.

By abuse of notation we call the varieties $W_{t}$ Del Pezzo 3-folds. Their normalization have only isolated double points from the contracted lines between basepoints, these occur when $t \leq 6$. For each $t$ we want to describe the ideal of $W_{t}$ and understand their linkage class.

Proposition 4.5. A 3-fold $W_{5}$ is bilinked to the union of two $\mathbf{P}^{3}$ 's which span $\mathbf{P}^{5}$. A 3-fold $W_{6}$ is bilinked to the union of three $\mathbf{P}^{3}$ 's. The ideals of general Del 
Pezzo 3-folds $W_{7}$ and $W_{8}$ are generated by quartics and quintics, and quintics and sextics respectively.

Proof. To understand the ideal of $W_{t}$ we first investigate the ideal of the singular lines. For $t=5$ there is one line so this case is trivial. The case $t=6$ is also easy since there are three lines; they span $\mathbf{P}^{5}$ as soon as the projection is general, as is easily verified. For $t=7$ and $t=8$ the situation is a bit more involved.

Lemma 4.6. The 6 singular lines of a general Del Pezzo 3-fold $W_{7}$ lie in a determinental net of quadrics in $\mathbf{P}^{5}$, in fact in the Segre embedding of $\mathbf{P}^{1} \times \mathbf{P}^{2}$.

Proof. Let $V$ be the linear system of quadrics which define the rational map of $\mathbf{P}^{3}$ onto $W_{7}$. By Lemma 4.3 the linear system $V$ contains and is spanned by at least two determinental nets. Of course, the corresponding twisted cubic curves pass through the basepoint, so the union of any two is rational and is therefore contained in a cubic surface. By genericity we may assume that this surface is smooth. On this cubic surface there is a pencil of twisted cubic curves through the basepoint with six common secant lines. This pencil of curves corresponds to a linear pencil of nets of quadrics, so since two nets are contained in $V$ they all are. The images of these twisted cubic curves on $W_{7}$ are plane curves. Since the twisted cubic curves pass through the basepoint of $V$ these plane curves have degree 5 . The 6 common secant lines are mapped to the 6 singular lines on $W_{7}$ and they account for the 6 singular points on each of these plane rational quintic curves.

Consider the space of quadrics passing through the 6 singular lines. If any of these quadrics intersects a plane of the plane quintic curves properly, its intersection would be a conic section passing through the 6 singular points of the quintic curve. But this is impossible by Bezout. Therefore any quadric which passes through the 6 lines must contain the planes of these curves. The intersection of these quadrics is therefore at least a threefold. Now, 6 lines impose at most 18 conditions on quadrics, so there are at least 3 such quadrics and the intersection is a threefold. A codimension 2 variety contained in 3 quadrics is a rational cubic scroll. If this scroll is singular, it is a cone, and any two planes meet. In this case two planes span a hyperplane. Pulled back to the cubic surface of the twisted cubic curves, this hyperplane corresponds to a quadric which contains the two curves. But the union of the two curves has arithmetic genus 0 , so it is not contained in a quadric and a cubic surface. Therefore the scroll is smooth, isomorphic to the Segre 3-fold scroll.

Proposition 4.7. $W_{7}$ is contained in five quartic hypersurfaces, they define an arithmetically Cohen Macaulay 3 -fold, the union of $W_{7}$ and the rational cubic scroll $R$ which contains the singular lines of $W_{7}$. The quartic hypersurfaces are defined by the maximal minors of a $4 \times 5$ matrix with linear entries.

Proof. Again let $V$ be the linear system of quadrics which define the rational map of $\mathbf{P}^{3}$ onto $W_{7}$. In the proof of Lemma 4.6 we saw that there is a pencil of twisted 
cubic curves through the basepoint $p$ of $V$ whose defining nets of quadrics are contained in $V$. These twisted cubic curves sweep out a cubic surface $S_{3}$ whose image contains the singular lines on $W_{7}$ and is contained in the rational cubic scroll $R$. The linear system of quadrics $V$ restricts to $S_{3}$ with one base point, so the image has degree 11. Consider the quartic surfaces through $S_{3}$ and singular in $p$; they consist of the union of $S_{3}$ and planes through $p$, so these quartics form a net. On the other hand, the image of $S_{3}$ in $\mathbf{P}^{5}$ is contained in the net of quadrics through $R$, and these quadrics pulled back to $\mathbf{P}^{3}$, correspond to quartic surfaces through $S_{3}$ singular at $p$, i.e. to the above net of quartics. Since the net of quartics has no unassigned basepoints, the quadrics through $R$ define precisely the image of $S_{3}$ on $W_{7}$, i.e. the intersection $W_{7} \cap R$ is precisely the image of the surface $S_{3}$. The union $W_{7} \cup R$ has degree 10 and genus 11 . To conclude that the union is arithmetically Cohen Macaulay we give an example. Let $V$ be the space of quadrics

$$
\left\langle x_{0}^{2}+x_{1} x_{2}, x_{1}^{2}+x_{2} x_{3}, x_{2}^{2}+x_{3} x_{0}, x_{0} x_{1}, x_{0} x_{1}+x_{2} x_{3}, x_{0} x_{2}+x_{1} x_{3}\right\rangle .
$$

A straightforward computation in [MAC] shows that the Del Pezzo 3-fold $W_{7}$ defined by $V$ lies in precisely 5 quartics, the $4 \times 4$ minors of a $4 \times 5$ matrix with linear entries, i.e. these quartics define an arithmetically Cohen Macaulay scheme of degree 10 and genus 11. Since this is an open condition in the Hilbert scheme [Ell], the same is true for the general projection $W_{7}$.

For $W_{8}$ we get somewhat less.

Lemma 4.8. $W_{8}$ contains two plane rational sextic curves, and each of the singular lines is spanned by a pair of nodes of these two sextic curves. In particular there are sextic generators in the ideal of $W_{8}$.

Proof. In this case there are two apolar twisted cubic curves, these are mapped to plane sextic curves with 10 double points at the intersection of these planes with the 10 singular lines of $W_{8}$. So we need sextic generators in the ideal of $W_{8}$.

We are now ready to give some numerical results for the ideals of general Del Pezzo 3 -folds $W_{t}$ of degree $5 \leq t \leq 8$.

Lemma 4.9. Table 3 gives the degrees $d$ and the number of generators in the ideal of $W_{t}$, for $t=5,6,7,8$.

Table 3.

\begin{tabular}{cccccc}
\hline & $d=2$ & $d=3$ & $d=4$ & $d=5$ & $d=6$ \\
\hline$t=5$ & 0 & 5 & 0 & 0 & 0 \\
$t=6$ & 0 & 1 & 7 & 0 & 0 \\
$t=7$ & 0 & 0 & 5 & 5 & 0 \\
$t=8$ & 0 & 0 & 1 & 10 & $\geq 1$ \\
\hline
\end{tabular}


Proof. The following spaces $V$ of quadrics

$$
\begin{gathered}
\left\langle x_{1} x_{2}+x_{0} x_{3}, x_{3}^{2}+x_{2} x_{0}, x_{3}^{2}+x_{1} x_{0}, x_{3}^{2}+x_{2} x_{1}, x_{0} x_{1}+x_{2} x_{3}, x_{0} x_{2}+x_{1} x_{3}\right\rangle, \\
\left\langle x_{0}^{2}+x_{1} x_{2}, x_{1}^{2}+x_{2} x_{3}, x_{3} x_{0}, x_{0} x_{1}, x_{0} x_{1}+x_{2} x_{3}, x_{0} x_{2}+x_{1} x_{3}\right\rangle, \\
\left\langle x_{0}^{2}+x_{1} x_{2}, x_{1}^{2}+x_{2} x_{3}, x_{2}^{2}+x_{3} x_{0}, x_{0} x_{1}, x_{0} x_{1}+x_{2} x_{3}, x_{0} x_{2}+x_{1} x_{3}\right\rangle,
\end{gathered}
$$

and

$$
\left\langle x_{0}^{2}+x_{1} x_{2}, x_{1}^{2}+x_{2} x_{3}, x_{2}^{2}+x_{3} x_{0}, x_{3}^{2}+x_{0} x_{1}, x_{0} x_{1}+x_{2} x_{3}, x_{0} x_{2}+x_{1} x_{3}\right\rangle,
$$

have respectively $3,2,1$ and no basepoints. The ideals of the corresponding Del Pezzo 3 -folds in $\mathbf{P}^{5}$ are easily computed in [MAC] and have Betti numbers as in the table.

For the proof of the lemma we show that the table represents a lower bound on the number of generators in each of the given degrees. The lemma then follows by semicontinuity.

In each case we define $\Sigma$ to be the union of the singular lines $L_{i}$ on $W_{t}$, i.e. precisely the non-normal double point locus of $W_{t}$. Let $V_{t}$ be the normalization of $W_{t}$, i.e. $V_{t}$ is isomorphic to $\mathbf{P}^{3}$ blown up in $8-t$ points, and with $\left(\begin{array}{c}8-t \\ 2\end{array}\right)$ lines contrated to ordinary double points. The map $\varphi: V_{t} \rightarrow W_{t}$ is double precisely along $\Sigma$, thus we get an exact sequence of sheaves

$$
0 \rightarrow \mathcal{O}_{W_{t}} \rightarrow \varphi_{*} \mathcal{O}_{V_{t}} \rightarrow \mathcal{O}_{\Sigma}(-1) \rightarrow 0
$$

where $\mathcal{O}_{\Sigma}=\oplus_{i=1}^{l} \mathcal{O}_{L_{i}}$ and $l=\left(\begin{array}{c}t-3 \\ 2\end{array}\right)$. Since $V_{t}$ is a Del Pezzo 3-fold, the cohomology of this sequence gives $\mathrm{h}^{3}\left(\mathcal{O}_{W_{t}}(k)\right)=\mathrm{h}^{3}\left(\varphi_{*} \mathcal{O}_{V_{t}}(k)\right)=0$, when $k \geq 3$. Furthermore the Euler characteristic of the relevant twists of the ideal sheaf of $W_{t}$ is easily computed from this exact sequence together with the exact sequence

$$
0 \rightarrow \mathcal{I}_{W_{t}}(k) \rightarrow \mathcal{O}_{\mathbf{P}^{5}}(k) \rightarrow \mathcal{O}_{W_{t}}(k) \rightarrow 0 .
$$

We collect the results in the following table.

Table 4.

\begin{tabular}{ccccccc}
\hline & $k$ & $\chi\left(\mathcal{O}_{W_{t}}(k)\right)$ & $\mathrm{h}^{0}\left(\varphi_{*} \mathcal{O}_{V_{t}}(k)\right)$ & $h^{0}\left(\mathcal{O}_{\Sigma}(k-1)\right)$ & $\mathrm{h}^{0}\left(\mathcal{O}_{\mathbf{P}^{5}}(k)\right)$ & $\chi\left(\mathcal{I}_{W_{t}}(k)\right)$ \\
\hline$t=5$ & & & 54 & 3 & 56 & 5 \\
& 3 & 51 & 54 & 9 & 56 & 1 \\
& & & 55 & 12 & 126 & 13 \\
& 4 & 113 & 125 & & & \\
$t=7$ & & & & 24 & 126 & 5 \\
& 4 & 121 & 145 & 30 & 252 & 31 \\
& 5 & 221 & 251 & & & \\
& & & & 40 & 126 & 1 \\
& 4 & 125 & 165 & 50 & 252 & 16 \\
\hline
\end{tabular}


By restriction to general $\mathbf{P}^{3}$ sections of $W_{t}$ it is easy to check that $\mathrm{h}^{1}\left(\mathcal{O}_{W_{t}}(k)\right)=$ 0 for the values of $k$ in the table. Since additionally $\mathrm{h}^{3}\left(\mathcal{O}_{W_{t}}(k)\right)=0$ when $k \geq 3$, the Euler characteristic of the twisted ideal is a lower bound for $\mathrm{h}^{0}\left(\mathcal{I}_{W_{t}}(k)\right)$. Thus the Betti numbers of the ideal follow except in the case of quintics and $W_{7}$ and sextics and $W_{8}$. These cases are accounted for in Proposition 4.7 and Lemma 4.8. In fact, from Lemma 4.7 we get 4 linear syzygies among the 5 quartics in the ideal of $W_{7}$. Therefore there are also 4 extra quintic generators in the ideal, 5 altogether. Lemma 4.8 says that there are sextic generators in the ideal of $W_{8}$.

To finish the proof of Proposition 4.5 it remains to consider the linkage classes of $W_{5}$ and $W_{6}$. The 3 -fold $W_{5}$ is linked $(3,3)$ to a rational 3 -fold scroll of degree 4. This lies in a quadric and is linked $(2,3)$ to two $\mathbf{P}^{3}$ 's, which clearly is minimal in its even biliaison class.

$W_{6}$ is linked $(3,4)$ to a 3 -fold $U$ with sectional genus 1 which lies on two cubics. Consider a $\mathbf{P}^{3}$ spanned by two singular lines in $W_{6}$. It intersects $W_{6}$ in the two lines and in two additional skew lines each intersecting both the singular lines. Clearly every cubic through $W_{6}$ contain this $\mathbf{P}^{3}$, so $U$ must intersect it in a quartic surface singular along the two singular lines. $U$ is therefore an elliptic scroll linked $(3,3)$ to the union of three $\mathbf{P}^{3}$ 's (cf. also section 6 ). This concludes the proof of Proposition 4.5 .

In the last section we need a converse to Proposition 4.7.

Proposition 4.10. Let $W$ be a 3-fold with sectional genus 1, with non-normal double points along 6 skew lines, no 3 in a $\mathbf{P}^{3}$ and not all 6 on a rational normal quartic scroll. Assume that there is a Segre cubic scroll $R$ in $\mathbf{P}^{5}$ containing the 6 skew lines transverse to its planes and intersecting $W$ in a surface of degree 11 , linked to 4 planes in the intersection of $R$ with a quintic hypersurface. Assume furthermore that $W \cup R$ is scheme-theoretically defined by the $4 \times 4$ minors of a $4 \times 5$ matrix with linear entries, and that the only common singularities of these quartics are the 6 lines. Then $W$ is a Del Pezzo 3-fold $W_{7}$.

Proof. Consider the $4 \times 5$ matrix $M$ whose maximal minors define $R \cup W$. Let $\left\langle z_{0}, \ldots, z_{3}\right\rangle$ be the coordinates of $\mathbf{P}^{3}$, then the 5 bilinear equations

$$
\left(z_{0}, z_{1}, z_{2}, z_{3}\right) \cdot M=(0,0,0,0,0)
$$

define a 3 -fold $T$ in $\mathbf{P}^{3} \times \mathbf{P}^{5}$. The projection to $\mathbf{P}^{5}$ is onto $W \cup R$, while the other projection is onto $\mathbf{P}^{3}$. The fiber in $T$ over any point in $\mathbf{P}^{3}$ is linear, defined by the 5 linear equations in the 6 coordinate functions of $\mathbf{P}^{5}$, so for the general point in $\mathbf{P}^{3}$ the fiber is a single point. The fiber over any point in $W \cup R$ is also linear, with dimension equal to 3 minus the rank of $M$ at the target point. The points where $M$ has rank at most 2, are singular on $W \cup R$, in fact they are singular on any quartic minor of $M$, so by our assumption, these points all lie on the 6 singular lines. Outside the 6 singular lines $M$ has rank 3 and therefore there is a rational 
map

$$
\varphi: W \cup R--->\mathbf{P}^{3}
$$

which is a morphism outside these 6 lines. This map is birational on one component of $W \cup R$ and contracts every other component to a surface, a curve or a point. The inverse map

$$
\psi: \mathbf{P}^{3}--->W \cup R
$$

is defined by the maximal minors of a $5 \times 6$-matrix $M^{\prime}$ with linear entries. $M^{\prime}$ is obtained from $M$ by interchanging rows and linear forms. Surfaces that are images of components of $W \cup R$ are fixed components for the linear system of quintic minors of $M^{\prime}$.

Our first aim is to show that $W$ has to be irreducible. We analyse carefully possible reducible components of the surface $S=W \cap R$. For this we start with the intersection of $S$ with a general plane in $R$.

On $R$ the surface $S \equiv 5 h-4 f$, where $h$ is the class of a hyperplane section, while $f$ is the class of a plane. Since $W$ is non-normal along 6 lines transverse to the planes of $R$, the intersection of $S$ with a general plane is a curve $C$ of degree 5 singular in 6 points, the points of intersection between the plane and the singular lines. Furthermore, no three singular lines span a $\mathbf{P}^{3}$, so no three of the singular points of $C$ are collinear. More generally, any effective divisor of type $a h-a f$ is the product of a plane curve of degree $a$ and $\mathbf{P}^{1}$. Geometrically each $\mathbf{P}^{1}$ is a line transverse to all planes in $R$. Conversely, any irreducible divisor of type $h+b f$ that contains more than one line transverse to all planes has $b=-1$ and is a quadric surface, while any irreducible divisor of type $2 h+b f$ that contains more than 4 lines transverse to all planes has $b=-2$ and is a rational normal quartic scroll. In our situation, this means that three singular lines intersect no plane in collinear points. Also, since not all 6 singular lines are on a rational normal quartic scroll, the 6 singular points cannot lie on a conic section. Therefore, if $C$ is reducible it is either the union of 2 conics and a line, or the union of a conic and a cubic. In the former case the singular points are the points of intersection between the two conics and one point of intersection between the line and each conic, while in the latter case 5 singular points are points of intersection between the conic and the cubic, while the last point is singular on the cubic.

Next, the surface $S$ intersects each general line in $R$ transverse to the planes in one point. Since the intersection of $S$ with a general plane has at most 3 components, $S$ itself has at most 4 components: Only the planes in $R$ do not intersect the general plane in a curve, so since exactly one component intersects every line transverse to the planes, there are at most 4 components of $S$.

To describe the possible components of $S$ we first note that on $R$ any effective divisor is of type $a h-b f$, with $a \geq 0$ and $b \leq a$. In our two cases of possible reducible curves in general planes we have the following possible decompositions of $S$ into irreducible components:

$$
S \equiv 5 h-4 f=b_{0} f+\left(h-b_{1} f\right)+\left(2 h-b_{2} f\right)+\left(2 h-b_{2}^{\prime} f\right)
$$


where $b_{1}+b_{2}+b_{2}^{\prime}-b_{0}=4$ and

$$
S \equiv 5 h-4 f=b_{0} f+\left(2 h-b_{2} f\right)+\left(3 h-b_{3} f\right)
$$

where $b_{2}+b_{3}-b_{0}=4$. With the above restriction on the $b_{i}$, the former case occurs only when $\left(b_{0}, b_{1}, b_{2}, b_{2}^{\prime}\right)=(1,1,2,2),\left(b_{0}, b_{1}, b_{2}, b_{2}^{\prime}\right)=(0,0,2,2)$ or $\left(b_{0}, b_{1}, b_{2}, b_{2}^{\prime}\right)=$ $(0,1,2,1)$. The latter case occurs only when $\left(b_{0}, b_{2}, b_{3}\right)=(1,2,3),\left(b_{0}, b_{2}, b_{3}\right)=$ $(0,2,2)$ or $\left(b_{0}, b_{2}, b_{3}\right)=(0,1,3)$.

We are now ready to analyse possible components of $W$. Clearly any two components of $W$ intersect each other along a surface or a curve (or both). If two components of $W \cup R$ intersect each other along a curve, then every hypersurface through $W \cup R$ has to be singular along this curve, so by our assumption this happens only along the 6 singular lines. The sectional genus of $W$ is 1 , thus for the general $\mathbf{P}^{3}$ section of $W$ the arithmetic genus is 1 plus the number of intersection points with the singular lines. This means that for a general $\mathbf{P}^{3}$ passing through a plane of $R$, the contribution to the arithmetic genus of the plane curve $C$ is 0 . The degree of $W$ is 7 , so residual to $C$ in this intersection is a curve of degree 2 . It must intersect $C$ in 2 points, and it must itself have arithmetic genus 0 , so it is a conic section, two connected lines or a double line. This very much restricts the possiblities for the irreducible components of $W$.

In our analysis of the components $W_{d}$ of $W$, we shall index them by their degree, unless the degree is not specified, in which case we use index 0 . This notation applies only in this proof and should not be confused with Del-Pezzo 3 -folds $W_{t}$. A component $W_{0}$ of $W$ must intersect $R$ in a curve or a surface. If it intersects $R$ only in a curve, then this curve meets the general plane in $R$ only in points, thus this component intersects a general $\mathbf{P}^{3}$ through a general plane in $R$ in a curve not contained in the plane. Therefore this component has degree 1 or 2. Similarly if $W_{0}$ intersects the general plane in $R$ in a curve of degree $a$, then the degree of the component is $a, a+1$ or $a+2$. In fact, if $W_{0}$ spans $\mathbf{P}^{5}$, i.e. when $a \geq 2$, then the degree is strictly greater than $a$. Of course, while $W_{0}$ is irreducible, $W_{0} \cap R$ may still be reducible. Anyway, we may enumerate the different cases in a table, where the columns are ordered by the degree of the curve of intersection between a component and a general plane, while the entries are the degrees of the components themselves:

Table 5.

\begin{tabular}{cccccc}
\hline 0 & 1 & 2 & 3 & 4 & 5 \\
\hline 1 & 0 & 0 & 0 & 0 & 6 \\
1 & 1 & 0 & 0 & 5 & 0 \\
0 & 1 & 0 & 0 & 0 & 6 \\
0 & 1 & 3,3 & 0 & 0 & 0 \\
0 & 0 & 3 & 4 & 0 & 0 \\
0 & 0 & 0 & 0 & 0 & 7 \\
\hline
\end{tabular}

In the first two cases of table 5 the first linear component $W_{1}$ may intersect $R$ in a plane and a line, or in a connected curve of degree 3 . The latter may be 
excluded since no connected cubic curve is supported on the 6 singular lines. In the former case the line must be one of the 6 singular lines. The remaining part of $W$ is a component of degree 6 or two components of degree 1 and 5 , respectively. These remaining components must intersect $R$ in a surface of type $5 h-5 f$, i.e. they do not intersect the general line in $R$ transverse to the planes. In the first case the linear component intersects the component $W_{6}$ of degree 6 along a quadric surface, since the sectional genus is 1 . The linear component $W_{1}$ intersects $R$ in a plane, but $W_{6}$ intersects the general plane in $R$ in a curve of degree 5 , so this plane must also be contained in $W_{6}$. This contradicts the fact that $W_{6}$ does not intersect the general line in $R$ transverse to the planes. The second case is impossible for the same reason.

In the third case of the table there is a linear component $W_{1}$ that intersects $R$ along a quadric surface and a component $W_{6}$ that intersects $R$ in a surface of type $4 h-3 f$. Again the two components have also to intersect along a quadric surface. The intersection of the two components on $R$ is a curve of type $(h-f)(4 h-3 f)=$ $4 h^{2}-7 h f$, i.e. a curve of degree 5 . On the other hand on $W_{1}$ each of the two other components intersect along a quadric surface and two singular lines. This adds up to a curve of degree 6 in the intersection of all three components. This is a contradiction. The fourth case is entirely similar.

In the fifth case there are two components $W_{3}$ and $W_{4}$ of degree 3 and 4 respectively. They intersect each other in a surface of degree 2 . Furthermore they intersect on $R$ in a curve of type $(2 h-f)(3 h-2 f)=6 h^{2}-7 h f$, i.e. a curve of degree 11. The union of the 5 singular lines in this intersection is a curve of type $5 h^{2}-10 h f$, so the remaining part is of type $h^{2}+3 h f$. This is a curve of degree 6. Again the surface $W_{3} \cap W_{4}$ has degree 2. If it is irreducible, then the lines of one of the rulings are at least 3 -secants to $R$, impossible, so the quadric surface is contained in $R$, again contradicting our assumption. If the surface $W_{3} \cap W_{4}$ is the union of two planes, then at least one of the planes intersects $R$ in a curve of degree at least 3 , again contradicting our assumption, so this case is also impossible. This concludes the proof that $W$ is irreducible.

It remains to show that $R$ is contracted by $\varphi$. If not, $W$ must be contracted. First of all this is possible only if $W$ is swept out by lines, i.e. has a line through every point. For this consider a general plane in $R$. It intersects $W$ in a plane quintic curve with 6 double points. $W$ has degree 7 , so in a general $\mathbf{P}^{3}$ through the plane $W$ has a curve of degree 2 residual to the plane curve. Since the arithmetic genus of $W$ is 1 and the plane curve is rational, this residual curve is a conic or two connected lines. Now, $W$ can only have a line through each point if this residual curve is always two lines. On the other hand if these two lines are contracted by $\varphi$, they are contracted to the same point, since the matrix $M$ has rank 3 even in the point of intersection. But all fibers of $\varphi$ are linear, so $W$ must contain a plane through the two lines, this is absurd. Thus $W$ is mapped birationally to $\mathbf{P}^{3}$ by $\varphi$.

It follows that the restriction of $\varphi$ to $R$ is a contraction onto a surface. Clearly it must contract the lines transverse to the planes. From the matrix $M$ we see that the map is defined by cubics on each plane, and it is birational, so the image has 
degree at least 3 . Therefore, the inverse map is defined by hyperplanes or quadrics. Since $W$ has degree 7 the inverse map must be defined by quadrics, and in fact with one basepoint, i.e. $W$ is a Del Pezzo 3 -fold $W_{7}$.

\section{Non-normal Calabi-Yau 3-folds in $\mathbf{P}^{5}$}

We shall show that $W_{t}$ for $t=5,6,7$ is bilinked on a quintic hypersurface to a non-normal Calabi-Yau 3-fold. $W_{t}$ is assumed to be general in the sense of section 4 , including in the case of $W_{7}$ any Del Pezzo 3 -fold characterized as in Proposition 4.10. Most of the argument goes through also for $W_{8}$, but since we shall not need this later we do not conclude in this case (see Remark 5.3).

Lemma 5.1. The general Del Pezzo 3 -fold $W_{t}$ for $t=5,6,7,8$ is contained in an irreducible quintic hypersurface. The general such quintic hypersurface is normal, it has double points along the singular lines of $W_{t}$ and has only canonical singularities.

Proof. We start with a general $W_{t}$ and a general quintic hypersurface $Q$ through $W_{t}$. Since $W_{t}$ has non-normal double points along the singular lines and quintics generate its ideal, $Q$ has multiplicity 2 at a general point on a singular line. Let

$$
p^{\prime}: \mathrm{Bl}_{s}\left(\mathbf{P}^{5}\right) \rightarrow \mathbf{P}^{5}
$$

be the blowup of $\mathbf{P}^{5}$ along the singular lines of $W_{t}$, and denote by $W_{t}^{\prime}$ and $Q^{\prime}$ the strict transforms of $W_{t}$ and $Q$. Then $W_{t}^{\prime}$ has at most ordinary double points as singularities.

Next we consider the blowup

$$
p^{\prime \prime}: \mathrm{Bl}_{s, W}\left(\mathbf{P}^{5}\right) \rightarrow \mathrm{Bl}_{s}\left(\mathbf{P}^{5}\right)
$$

of $\mathrm{Bl}_{s}\left(\mathbf{P}^{5}\right)$ along $W_{t}^{\prime}$. Over the singular points of $W_{t}^{\prime}$, the blowup $\mathrm{Bl}_{s, W}\left(\mathbf{P}^{5}\right)$ will have isolated double points. Denote by $Q^{\prime \prime}$ the strict transform of $Q^{\prime}$ and let $W_{t}^{\prime \prime}$ be the strict transform of $W_{t}^{\prime}$ in $Q^{\prime \prime}$. By the fundamental property of blowup, $W_{t}^{\prime \prime}$ is a Cartier divisor on $Q^{\prime \prime}$.

We now analyse the situation, assuming that we have chosen $Q$ general. For $t \leq 7$, the ideal of $W_{t}$ is generated by quintics by Proposition 4.5, so in this case $Q^{\prime \prime}$ is smooth outside $W_{t}^{\prime \prime}$. We claim that $Q^{\prime \prime}$ is smooth and that $p^{\prime \prime}$ restricted to $Q^{\prime \prime}$ is a small resolution of $Q^{\prime}$. Since the quintics generate the ideal of $W_{t}$, the conormal sheaf of $W_{t}^{\prime}$ in $\mathrm{Bl}_{s}\left(\mathbf{P}^{5}\right)$ twisted by the class of $Q^{\prime}$ is generated by its global sections. In particular, any divisor equivalent to $Q^{\prime}$ induces a section of this sheaf, which is a rank 2 bundle outside the singular points. Its zero locus is the subscheme of $W_{t}^{\prime}$ defined by the Jacobian ideal of $Q^{\prime}$. For general $Q$ this is a smooth curve which does not pass through the singular points of $W_{t}^{\prime}$. Thus $Q^{\prime}$ is singular only along some smooth curve on $W_{t}^{\prime}$ not passing through the singularities of $W_{t}^{\prime}$. The map $p^{\prime \prime}$ defines an isomorphism between $W_{t}^{\prime \prime}$ and $W_{t}^{\prime}$. Moreover since $W_{t}^{\prime \prime}$ is a Cartier 
divisor on $Q^{\prime \prime}$ it follows that $Q^{\prime \prime}$ is smooth and that $p^{\prime \prime}$ defines a small resolution of $Q^{\prime}$ showing that $Q^{\prime}$ has only canonical singularities. For $W_{8}$ one may show with [MAC] in the example of Lemma 4.9 that the baselocus of the quintics through $W_{8}$ is precisely the singular lines and the two planes. Therefore also in this case $Q^{\prime \prime}$ is smooth and we can argue as before.

Finally $Q$ is smooth in codimension 1 and since it is a hypersurface, it is normal. It remains to prove that $Q$ has canonical singularities along the singular lines. First recall that $Q$ has multiplicity 2 at the generic point of the singular lines. Moreover the blowup $p^{\prime}$ defines a resolution of $Q$ over the points on the singular lines outside the singular curve of $Q^{\prime}$. Hence $Q$ has transversal ordinary double points outside the finitely many points on the singular lines which are the intersection with the image under $p^{\prime}$ of the singular curve of $Q^{\prime}$. In particular $Q$ has canonical singularities outside these finitely many points. Combining this with the argument that $p^{\prime \prime}$ defines a small resolution of $Q^{\prime}$ gives the claim of the lemma.

Proposition 5.2. The general Del Pezzo 3-fold $W_{t}$ for $t=5,6,7$ is bilinked $(5,4)$ and $(5,5)$ on a quintic hypersurface to a variety $Y$ of degree $t+5$ which has nonnormal singularities along the singular lines of $W_{t}$. The normalization of $Y$ is a smooth Calabi-Yau 3-fold.

Proof. We start with a general quintic hypersurface $Q$ which contains $W_{t}$ as in Lemma 5.1, and keep the same setup and notation as in the above proof. In addition we let $H_{Q^{\prime}}$ and $H_{Q^{\prime \prime}}$ be the pullback by $p^{\prime}$ and $p^{\prime \prime}$ of a hyperplane $H$ restricted to $Q$. A 3-fold $Y$ bilinked in hypersurfaces of degree 4 and 5 to $W_{t}$ on $Q$ is nothing but a Weil divisor equivalent to $W_{t}+H_{Q}$, where $H_{Q}$ is the restriction of $H$ to $Q$. The strict transform $Y^{\prime \prime}$ of $Y$ on $Q^{\prime \prime}$ is a Cartier divisor linearly equivalent to $W_{t}^{\prime \prime}+H_{Q^{\prime \prime}}$. To analyze the singularities and the canonical sheaf of $Y$ we perform adjunction on the smooth 4 -fold $Q^{\prime \prime}$.

Let $E^{\prime \prime}$ be the pullback of the exceptional divisor of $\mathrm{Bl}_{s}\left(\mathbf{P}^{5}\right)$ to $\mathrm{Bl}_{s, W}\left(\mathbf{P}^{5}\right)$. Then the canonical line bundle on $Q^{\prime \prime}$ is $\mathcal{O}_{Q^{\prime \prime}}\left(E^{\prime \prime}-H_{Q^{\prime \prime}}\right)$. By adjunction on $Q^{\prime \prime}$, the canonical line bundle on $W_{t}^{\prime \prime}$ is $\mathcal{O}_{W_{t}^{\prime \prime}}\left(W_{t}^{\prime \prime}+E^{\prime \prime}-H_{Q^{\prime \prime}}\right)$. Consider now the normalization $\tilde{W}_{t}$ of $W_{t}$. The map $W_{t}^{\prime \prime} \rightarrow W_{t}$ factors through this normalization. On $\tilde{W}_{t}$ there are no exceptional divisors since the singular locus has codimension 2. Therefore, the map $W_{t}^{\prime \prime} \rightarrow \tilde{W}_{t}$ is the blowup along a curve with exceptional line bundle $\mathcal{O}_{W_{t}^{\prime \prime}}\left(E^{\prime \prime}\right)$. But $\tilde{W}_{t}$ is a Del Pezzo 3 -fold with only canonical Gorenstein singularities, so the canonical divisor of $W_{t}^{\prime \prime}$ is also $\mathcal{O}_{W_{t}^{\prime \prime}}\left(E^{\prime \prime}-2 H_{Q^{\prime \prime}}\right)$. Therefore $\mathcal{O}_{W_{t}^{\prime \prime}}\left(W_{t}^{\prime \prime}-H_{Q^{\prime \prime}}\right)=\mathcal{O}_{W_{t}^{\prime \prime}}\left(-2 H_{Q^{\prime \prime}}\right)$ and we obtain the equality

$$
\mathcal{O}_{W_{t}^{\prime \prime}}\left(W_{t}^{\prime \prime}+H_{Q^{\prime \prime}}\right)=\mathcal{O}_{W_{t}^{\prime \prime}}
$$

Now, consider the exact sequences of sheaves on $Q^{\prime \prime}$

$$
\begin{gathered}
0 \longrightarrow \mathcal{O}_{Q^{\prime \prime}}\left(H_{Q^{\prime \prime}}\right) \longrightarrow \mathcal{O}_{Q^{\prime \prime}}\left(W_{t}^{\prime \prime}+H_{Q^{\prime \prime}}\right) \longrightarrow \mathcal{O}_{W_{t}^{\prime \prime}}\left(W_{t}^{\prime \prime}+H_{Q^{\prime \prime}}\right) \longrightarrow 0 \\
0 \longrightarrow \mathcal{O}_{Q^{\prime \prime}} \longrightarrow \mathcal{O}_{Q^{\prime \prime}}\left(H_{Q^{\prime \prime}}\right) \longrightarrow \mathcal{O}_{H_{Q^{\prime \prime}}}\left(H_{Q^{\prime \prime}}\right) \longrightarrow 0
\end{gathered}
$$


and

$$
0 \longrightarrow \mathcal{O}_{H_{Q^{\prime \prime}}} \longrightarrow \mathcal{O}_{H_{Q^{\prime \prime}}}\left(H_{Q^{\prime \prime}}\right) \longrightarrow \mathcal{O}_{H_{Q^{\prime \prime}} \cap H^{\prime}{ }_{Q^{\prime \prime}}}\left(H_{Q^{\prime \prime}}\right) \longrightarrow 0
$$

for general hyperplanes $H$ and $H^{\prime}$. Since $Q$ and $Q \cap H$ have only canonical singularities, $Q^{\prime \prime}$ and $H_{Q^{\prime \prime}}$ are regular. Thus the second and third sequence remain exact on global sections. Furthermore $H \cap H^{\prime} \cap Q$ is a smooth quintic surface, so $\mathrm{h}^{1}\left(\mathcal{O}_{H \cap H^{\prime} \cap Q}(H)\right)=\mathrm{h}^{1}\left(\mathcal{O}_{H_{Q^{\prime \prime}} \cap H^{\prime}{ }_{Q^{\prime \prime}}}\left(H_{Q^{\prime \prime}}\right)\right)=0$. Therefore $\mathrm{h}^{1}\left(\mathcal{O}_{H_{Q^{\prime \prime}}}\left(H_{Q^{\prime \prime}}\right)\right)=$ $\mathrm{h}^{1}\left(\mathcal{O}_{Q^{\prime \prime}}\left(H_{Q^{\prime \prime}}\right)\right)=0$ and also the first sequence remains exact after taking global sections. Thus the line bundle in the middle is generated by global sections, i.e. the linear system $\left|W_{t}^{\prime \prime}+H_{Q^{\prime \prime}}\right|$ of divisors on $Q^{\prime \prime}$ has no basepoints. By Bertini, we may conclude that a general member $Y^{\prime \prime}$ of this linear system is smooth.

The exceptional locus of $p^{\prime \prime}$ on $Q^{\prime \prime}$ is a surface scroll, and $W_{t}^{\prime \prime}$ intersects this scroll in a section over the smooth base curve. The restriction of the linear system $\left|W_{t}^{\prime \prime}+H_{Q^{\prime \prime}}\right|$ to this scroll has no basepoints so the general member is again a section over the base curve. Therefore the restriction of $p^{\prime \prime}$ to $Y^{\prime \prime}$ is also an isomorphism, and the image $Y^{\prime}$ on $Q^{\prime}$ is smooth, while $Y$ is singular only along the singular lines. Since $W_{t}$ has non-normal double points along the singular lines, the same is the case for $Y$.

The canonical line bundle on $Y^{\prime \prime}$ is easily computed by adjunction on $Q^{\prime \prime}$. In fact it is

$$
\mathcal{O}_{Y^{\prime \prime}}\left(Y^{\prime \prime}+E^{\prime \prime}-H_{Q^{\prime \prime}}\right)=\mathcal{O}_{Y^{\prime \prime}}\left(W_{t}^{\prime \prime}+E^{\prime \prime}\right) .
$$

Since $\mathcal{O}_{W_{t}^{\prime \prime}}\left(Y^{\prime \prime}\right)=\mathcal{O}_{W_{t}^{\prime \prime}}\left(W_{t}^{\prime \prime}+H_{Q^{\prime \prime}}\right)=\mathcal{O}_{W_{t \prime}^{\prime \prime}}$, we have $\mathcal{O}_{Y^{\prime \prime}}\left(W_{t}^{\prime \prime}\right)=\mathcal{O}_{Y^{\prime \prime}}$. Therefore the canonical line bundle on $Y^{\prime \prime}$ is $\mathcal{O}_{Y^{\prime \prime}}\left(E^{\prime \prime}\right)$.

We turn to the singular variety $Y$. It is singular precisely along the singular lines of $W_{t}$ where it has non-normal double points. Consider the normalization $\tilde{Y} \rightarrow Y$. As in the case of $W_{t}$, the resolution of singularities $Y^{\prime \prime} \rightarrow Y$ factors through this normalization. We will show that $\tilde{Y}$ is already smooth. For this we first specialize the quintic hypersurface $Q$ to a hypersurface containing $W_{t}$ and a 3 -fold $Z$ that is smooth along the singular lines of $W_{t}$. In case $t=7$, we take $Z$ to be the Segre cubic scroll $R$, while in the cases $t=5$ and $t=6$ we may take $Z$ to be one and two $\mathbf{P}^{3}$ 's respectively. We make the computation explicit in the case when $t=7$, which we will use later. The other cases are similar. Assume that $Q$ is a quintic which contains $W_{t}$ and the rational cubic scroll $R$. Let $Q_{0} \rightarrow Q$ be the blow up of $Q$ along $R$. This defines a small resolution of the singularities of $Q$ along the lines. Over each line there is an exceptional scroll. This scroll is isomorphic to some Hirzebruch surface $F_{a}$ for some $a \geq 0$. The strict transform of $W_{t}$ meets this scroll in a rational curve which is a bisection on the scroll, since $W_{t}$ is double along the line. Since $Y$ as a Weil divisor is equivalent to $W_{t}+H_{Q}$ on $Q$, and the strict transform of $H_{Q}$ intersects the scroll in a ruling, we get that the strict transform of $Y$ meets the exceptional scroll in a bisection which is an elliptic curve. The normalization of $Y$ factors through this small resolution and, therefore, has elliptic curves lying over the singular lines. In particular the normalization is smooth over the singular lines. By deformation to the general quintic $Q$, the normalization of $Y$ is smooth. 
Now, the normalization $\tilde{Y}$ of $Y$ is isomorphic to $Y$ in codimension 1, therefore the canonical line bundle $\mathcal{O}_{Y^{\prime \prime}}\left(E^{\prime \prime}\right)$ on $Y^{\prime \prime}$ is the exceptional line bundle of the map $Y^{\prime \prime} \rightarrow \tilde{Y}$ and the canonical line bundle of $\tilde{Y}$ is trivial.

The irregularity of $\tilde{Y}$ equals the irregularity of $Y^{\prime \prime}$, since they are birational and both are smooth. But on $Y^{\prime \prime}$ we get

$$
\mathrm{h}^{1}\left(\mathcal{O}_{Y^{\prime \prime}}\right)=\mathrm{h}^{2}\left(\mathcal{O}_{Q^{\prime \prime}}\left(-W_{t}^{\prime \prime}-H_{Q^{\prime \prime}}\right)\right)=\mathrm{h}^{1}\left(\mathcal{O}_{W_{t}^{\prime \prime}}\left(-H_{Q^{\prime \prime}}\right)\right)=0
$$

so the normalization of $Y$ is regular. Thus the normalization of $Y$ is a smooth Calabi-Yau 3-fold and the proposition follows.

Remark 5.3. The above proof goes through also for $W_{8}$, except for the question of a smooth normalization.

\section{Equations of elliptic scrolls in $\mathbf{P}^{5}$}

In the remaining sections we prove that the union of two elliptic scrolls which intersect along an abelian surface is bilinked to a Del Pezzo 3 -fold $W_{7}$. On the way we describe the family of these reducible Calabi-Yau 3-folds using Heisenberg symmetry. But first we study the ideal of elliptic scrolls without using this symmetry.

Recall from section 3 that two elliptic scrolls whose intersection is a $(1,6)$ polarized abelian surface in $\mathbf{P}^{5}$, are each singular along three lines. Therefore we restrict our attention to this kind of elliptic scrolls. We start with a lemma which gives a quick construction of such scrolls.

Proposition 6.1. The union of three $\mathbf{P}^{3}$ 's in $\mathbf{P}^{5}$, which meet pairwise in lines, is linked $(3,3)$ to an elliptic scroll. Furthermore any elliptic 3 -fold scroll of degree 6 , singular along three lines which span $\mathbf{P}^{5}$, is linked $(3,3)$ to three $\mathbf{P}^{3}$ 's.

Proof. First, consider three $\mathbf{P}^{3}$ 's which meet pairwise in lines and two general cubic hypersurfaces containing them. The linked variety $X$ has degree 6 and sectional genus 1 (cf. $[\mathrm{PS}]$ ). Since the complete intersection has trivial canonical bundle, each component intersects the rest along an anticanonical divisor. Therefore $X$ meets each of the $\mathbf{P}^{3}$ 's in a quartic surface, singular along the two lines of intersection with the other two $\mathbf{P}^{3}$ 's. These quartic surfaces clearly are elliptic scrolls: Through every point on the surface outside the two singular lines there is a unique line in the surface through the point intersecting the two lines, by Bezout, so the quartic surface is a scroll of lines. Through every point on the singular lines there are two rulings of the scroll. In the Grassmannian of lines the curve parametrizing the rulings has a double cover to each of the two singular lines, so the curve is of type $(2,2)$ on $\mathbf{P}^{1} \times \mathbf{P}^{1}$, i.e. it is elliptic. The normalization of $X$ therefore contains elliptic scrolls in parts of hyperplane sections. On the other hand, residual to each quartic surface in hyperplane sections, there is a pencil of surfaces of degree two on 
$X$. Residual to the $\mathbf{P}^{3}$ they form part of a complete intersection of two quadrics in the hyperplane. The other two $\mathbf{P}^{3}$ 's intersect this hyperplane in two planes passing through a point, so the surface of degree two on $X$ must be two planes residual to these in the complete intersection. This displays the scroll structure of $X$. Clearly the scroll is elliptic.

Next, consider an elliptic scroll $X_{F}$ of degree 6 and singular along three lines that span $\mathbf{P}^{5}$. Let

$$
\varphi: \mathbf{P}(\mathcal{E}) \rightarrow X_{F}
$$

be the normalization map. Like in section 3 the three lines correspond to a decomposition

$$
\mathcal{E}=\mathcal{L}_{0} \oplus \mathcal{L}_{1} \oplus \mathcal{L}_{2}
$$

where the $\mathcal{L}_{i}$ are line bundles of degree 2 . We have

$$
\mathrm{H}^{0}(F, \mathcal{E}) \cong \mathrm{H}^{0}\left(X_{F}, \mathcal{O}_{X_{F}}(1)\right) \cong \mathrm{H}^{0}\left(\mathbf{P}^{5}, \mathcal{O}_{\mathbf{P}^{5}}(1)\right)
$$

since the linear system that maps $\mathbf{P}(\mathcal{E})$ into $\mathbf{P}^{5}$ is complete. There is an isomorphism

$$
\mathrm{H}^{0}\left(\mathcal{O}_{\mathbf{P}(\mathcal{E})}(n)\right) \cong \mathrm{H}^{0}\left(F, \mathrm{~S}^{n} \mathcal{E}\right) \cong \mathrm{H}^{0}\left(F, \mathcal{L}_{0}^{n} \oplus \mathcal{L}_{0}^{n-1} \otimes \mathcal{L}_{1} \oplus \ldots \oplus \mathcal{L}_{2}^{n}\right)
$$

where the number of summands is

$$
\left(\begin{array}{c}
n+2 \\
2
\end{array}\right)=\frac{(n+1)(n+2)}{2}
$$

and the degree of each summand is $2 n$. This shows that

$$
h^{0}\left(\mathcal{O}_{\mathbf{P}(\mathcal{E})}(n)\right)=h^{0}\left(F, \mathrm{~S}^{n} \mathcal{E}\right)=n(n+1)(n+2) .
$$

We now compare this to the situation on the singular scroll $X_{F}$. Recall that there are three sections $F_{i} \subset \mathbf{P}(\mathcal{E})$ such that the map $\varphi$ restricted to $F_{i}$ induces a double cover $\varphi: F_{i} \rightarrow L_{i}$ onto a line $L_{i}$. This is branched over 4 points and hence

$$
\varphi_{*} \mathcal{O}_{F_{i}}=\mathcal{O}_{L_{i}} \oplus \mathcal{O}_{L_{i}}(-2),
$$

and we have the exact sequence

$$
0 \rightarrow \mathcal{O}_{L_{i}} \rightarrow \varphi_{*} \varphi^{*} \mathcal{O}_{L_{i}} \rightarrow \mathcal{O}_{L_{i}}(-2) \rightarrow 0
$$

where $\varphi_{*} \varphi^{*} \mathcal{O}_{L_{i}}=\varphi_{*} \mathcal{O}_{F_{i}}$.

Lemma 6.2. There is an exact sequence of sheaves

$$
0 \rightarrow \mathcal{O}_{X_{F}} \rightarrow \varphi_{*} \mathcal{O}_{\mathbf{P}(\mathcal{E})} \rightarrow \oplus_{i=0}^{2} \mathcal{O}_{L_{i}}(-2) \rightarrow 0
$$

Proof. The cokernel clearly has support on the locus on $X_{F}$ where the map is not an isomorphism, i.e. on the three lines $L_{i}$. Restricted to these lines the exact sequence reduces to the one above. Since the lines are disjoint, the cokernel is a direct sum. 
Lemma 6.3. After tensoring with $\mathcal{O}_{X_{F}}(n)$ for any nonnegative $n$ the sequence of Lemma 6.2 is exact on global sections.

Proof. For $n=0,1$ the statement is immediate, since the third term has no sections. Furthermore $\mathrm{h}^{1}\left(\mathcal{O}_{X_{F}}(1)\right)=\mathrm{h}^{1}\left(\mathcal{O}_{\mathbf{P}(\mathcal{E})}(1)\right)=0$ and $\mathrm{h}^{2}\left(\mathcal{O}_{X_{F}}(n)\right)=\mathrm{h}^{2}\left(\mathcal{O}_{\mathbf{P}(\mathcal{E})}(n)\right)=$ 0 for $n \geq 1$. Let $H$ be a general hyperplane, and $P=\mathbf{P}^{3}$ be a general 3 -space inside $H$. Then $X_{F} \cap P$ is a smooth elliptic curve, $\operatorname{so~}^{1}\left(\mathcal{O}_{X_{F} \cap P}(n)\right)=0$ for $n \geq 1$. Furthermore $\mathrm{h}^{1}\left(\mathcal{O}_{X_{F} \cap H}(1)\right)=0$. Inductively $\mathrm{h}^{1}\left(\mathcal{O}_{X_{F} \cap H}(n)\right) \leq \mathrm{h}^{1}\left(\mathcal{O}_{X_{F} \cap H}(n-\right.$ 1)) $=0$ for $n \geq 2$, and similarly

$$
\mathrm{h}^{1}\left(\mathcal{O}_{X_{F}}(n)\right) \leq \mathrm{h}^{1}\left(\mathcal{O}_{X_{F}}(n-1)\right)=0
$$

for $n \geq 2$ and the lemma follows.

Lemma 6.3 allows us to compute

$$
h^{0}\left(\mathcal{O}_{X_{F}}(n)\right)=n(n+1)(n+2)-3(n-1), \quad n \geq 1 .
$$

The natural maps $\mathrm{H}^{0}\left(\mathcal{O}_{\mathbf{P}^{5}}(n)\right) \rightarrow \mathrm{H}^{0}\left(\mathcal{O}_{X_{F}}(n)\right)$ and the dimension

$$
h^{0}\left(\mathcal{O}_{\mathbf{P}^{5}}(n)\right)=\operatorname{dim} \mathrm{S}^{n} \mathrm{H}^{0}(\mathcal{E})=\left(\begin{array}{c}
n+5 \\
5
\end{array}\right),
$$

gives us

$$
\mathrm{h}^{0}\left(\mathcal{I}_{X_{F}}(n)\right)-\mathrm{h}^{1}\left(\mathcal{I}_{X_{F}}(n)\right)=\left(\begin{array}{c}
n+5 \\
5
\end{array}\right)+3(n-1)-n(n+1)(n+2) .
$$

In particular $\mathrm{h}^{0}\left(\mathcal{I}_{X_{F}}(3)\right) \geq 2$. Clearly the complete intersection of two cubics through $X_{F}$ contains the $\mathbf{P}^{3}$ 's spanned by the pairs of singular lines, therefore $\mathrm{h}^{0}\left(\mathcal{I}_{X_{F}}(3)\right)=2$ and Proposition 6.1 follows.

Corollary 6.4. $\mathrm{h}^{0}\left(\mathcal{I}_{X_{F}}(5)\right)=54$.

Proof.

$$
\mathrm{h}^{0}\left(\mathcal{I}_{X_{F}}(5)\right)-\mathrm{h}^{1}\left(\mathcal{I}_{X_{F}}(5)\right)=54
$$

as follows from the computation in the proof of Proposition 6.1. But $X_{F} \cap \mathbf{P}^{2}$ for a general $\mathbf{P}^{2}$ is 4-regular in the sense of Castelnuovo-Mumford, so $\mathrm{h}^{1}\left(\mathcal{I}_{X_{F}}(n)\right)=0$ for $n \geq 3$.

\section{Heisenberg symmetry of elliptic scrolls in $\mathrm{P}^{5}$}

First we collect some basic observations. Recall the Heisenberg group of level 6:

$$
1 \rightarrow \mu_{6} \rightarrow H_{6} \rightarrow \mathbf{Z} / 6 \times \mathbf{Z} / 6 \rightarrow 0 .
$$

Given any subgroup $G \subset \mathbf{Z} / 6 \times \mathbf{Z} / 6$ we can consider the preimage

$$
H(G):=\pi^{-1}(G) \subset H_{6} .
$$


Let $\left\langle x_{0}, \ldots, x_{5}\right\rangle$ be a basis for a 6 -dimensional complex vector space $V$ and let $\left\langle e_{0}, \ldots, e_{5}\right\rangle$ be a basis for $V^{*}$. The Schrödinger representation $\rho: H \rightarrow G L(V, \mathbf{C})$ is defined by

$$
\sigma: x_{i} \mapsto x_{i+1}, \quad \tau: x_{i} \mapsto \rho^{i} x_{i}
$$

where $\rho=\rho=e^{2 \pi i / 6}$ and indices are taken modulo 6 . It defines, by restriction, representations

$$
\rho_{G}: H(G) \rightarrow G L(V, \mathbf{C}) .
$$

The group $\mathbf{Z} / 6 \times \mathbf{Z} / 6$ contains unique subgroups isomorphic to $\mathbf{Z} / 2 \times \mathbf{Z} / 2$, resp. $\mathbf{Z} / 3 \times \mathbf{Z} / 3$. The preimages of these groups in $H_{6}$ are isomorphic to the Heisenberg groups $\mathrm{H}_{2}$ and $\mathrm{H}_{3}$ of level 2 and 3 . Note, however, that in the case of $H_{3}$ the induced representation differs from the Schrödinger respresentation by the nontrivial automorphism of the Galois group.

Lemma 7.1. (i) There are 4 subgroups isomorphic to $\mathbf{Z} / 3$ in $\mathbf{Z} / 3 \times \mathbf{Z} / 3$.

(ii) There are 4 subgroups isomorphic to $\mathbf{Z} / 2 \times \mathbf{Z} / 6$ in $\mathbf{Z} / 6 \times \mathbf{Z} / 6$.

Proof. Claim (i) is trivial. Every subgroup isomorphic to $\mathbf{Z} / 2 \times \mathbf{Z} / 6$ is generated by the elements of order 2 and one element of order 3 in $\mathbf{Z} / 6 \times \mathbf{Z} / 6$, i.e. by the subgroup $\mathbf{Z} / 2 \times \mathbf{Z} / 2 \subset \mathbf{Z} / 6 \times \mathbf{Z} / 6$ and a subgroup of order 3 of the group $\mathbf{Z} / 3 \times \mathbf{Z} / 3 \subset \mathbf{Z} / 6 \times \mathbf{Z} / 6$. This shows (ii).

Remark 7.2. At the same time this gives us a natural $1: 1$ correspondence between the 4 groups of part (i) of Lemma 7.1 and the 4 groups of part (ii). We shall use this frequently in what follows.

We shall denote the 4 subgroups of $\mathbf{Z} / 6 \times \mathbf{Z} / 6$ which are isomorphic to $\mathbf{Z} / 2 \times \mathbf{Z} / 6$ by $K_{1}, \ldots, K_{4}$. For every group $G$ in $\mathbf{Z} / 6 \times \mathbf{Z} / 6$ we set

$$
H(G)_{\iota}=\langle H(G), \iota\rangle \subset G_{6} .
$$

We denote by $G_{2}$ resp. $G_{3}$ the groups $\left\langle H_{2}, \iota\right\rangle$, resp. $G_{3}=\left\langle H_{3}, \iota\right\rangle$. Next we need some elementary representation theory. We denote by $U$ the Schrödinger representation of $\mathrm{H}_{2}$.

Lemma 7.3. (i) As an $\mathrm{H}_{2}$-module $V \cong 3 U$,

(ii) as a $G_{2}$-module $V \cong 2 U_{+} \oplus U_{-}$, where $U_{+}$, resp. $U_{-}$means that $\iota$ acts by +1 , resp. -1 on $U$,

(iii) as $K_{i}$-modules $V \cong U \oplus U^{\prime} \oplus \bar{U}^{\prime}$. Here the subgroup of $K_{i}$ which is isomorphic to $\mathbf{Z} / 3$, acts on $U^{\prime}$ by a non-trivial character and on $\bar{U}^{\prime}$, by the inverse of this character. The involution ८ leaves $U$ fixed and interchanges $U^{\prime}$ and $\bar{U}^{\prime}$.

Proof. (i), (ii) We can decompose $V$ as an $H_{2}$-module as follows:

$$
\left\langle x_{0}, x_{3}\right\rangle,\left\langle x_{2}, x_{5}\right\rangle,\left\langle x_{4}, x_{1}\right\rangle .
$$

From this the claim is obvious. 
(iii) It is enough to consider one of the groups $K_{i}$. The others can be done in the same way, or one can use the normalizer $N_{6}$ of $H_{6}$ in $G L(6, \mathbf{C})$. Here we shall consider the subgroup $K$ given by $\tau^{2}$. One immediately checks that $\tau^{2}$ acts by $1, \rho^{4}, \rho^{2}\left(\rho=e^{2 \pi i / 6}\right)$ on the above submodules of $V$, hence giving the claim.

We next want to associate basic geometric objects in $\mathbf{P}^{5}$ to the subgroups $K_{i}, i=1,2,3,4$.

Lemma 7.4. (i) To every subgroup $H\left(K_{i}\right), i=1,2,3,4$ of $H_{6}$ one can associate a unique set of 3 lines in $\mathbf{P}^{5}$ which is an $H_{6}$-orbit such that $H\left(K_{i}\right)$ is the stabilizer of each of these lines. The distinguished subgroup of order 3 in $K_{i}$ fixes these lines pointwise.

(ii) Every $H_{6}$-orbit of lines in $\mathbf{P}^{5}$ consisting of 3 lines is one of the above.

Proof. Let $\left\{L_{1}, L_{2}, L_{3}\right\}$ be an $H_{6}$-orbit of lines in $\mathbf{P}^{5}$. Then every line $L_{j}$ has a stabilizer in $\mathbf{Z} / 6 \times \mathbf{Z} / 6$ of order 12 . This must then be one of the groups $K_{i}, i=$ $1,2,3,4$. Without loss of generality it suffices to consider the group generated by $\left\langle\tau, \sigma^{3}\right\rangle$. Then the action of $\tau$ on the vector space associated to such a line $L_{j}$ splits into a sum of two different characters. Therefore $L_{j}$ is spanned by two basis vectors $e_{k}, e_{l}$. To obtain invariance under $\sigma^{3}$ the only possibilities are $\left\langle e_{0}, e_{3}\right\rangle,\left\langle e_{1}, e_{4}\right\rangle$ and $\left\langle e_{2}, e_{5}\right\rangle$. Furthermore the distinguished subgroup order 3 generated by $\tau^{2}$ fixes these three lines pointwise.

Lemma 7.5. (i) To every subgroup $H\left(K_{i}\right), i=1,2,3,4$ of $H_{6}$ one can associate a unique set of three 3 -spaces in $\mathbf{P}^{5}$ which is an $H_{6}$-orbit such that $H\left(K_{i}\right)$ is the stabilizer of each of these $\mathbf{P}^{3^{\prime}}$ s.

(ii) Every $H_{6}$-orbit of 3-spaces in $\mathbf{P}^{5}$ consisting of three $\mathbf{P}^{3^{\prime}}$ s is one of the above.

Proof. This is the dual statement to Lemma 7.4. Given three lines $L_{1}, L_{2}, L_{3}$ which form an $H_{6}$-orbit, the three $\mathbf{P}^{3^{\prime}}$ s are the spaces spanned by two of these lines.

Next we turn to the space of cubic forms $\mathrm{H}^{0}\left(\mathcal{O}_{\mathbf{P}^{5}}(3)\right)$ which we want to study as an $H_{6^{-}}$, resp. $G_{6}$-module.

Lemma 7.6. The $G_{6}$-module $\mathrm{H}^{0}\left(\mathcal{O}_{\mathbf{P}^{5}}(3)\right)$ is a sum of four 2-dimensional and twelve 4-dimensional representations. As $\mathrm{H}_{3}$-representation it is a sum of characters. The trivial character corresponds to the four pencils. The other 8 come in pairs (given by the involution ८) and each pair determines three 4-dimensional irreducible $G_{6}$-representations. The subspace of 2-dimensional representations is spanned by

$$
\begin{gathered}
\left\langle x_{0}^{3}+x_{2}^{3}+x_{4}^{3}, x_{1}^{3}+x_{3}^{3}+x_{5}^{3}\right\rangle \\
\left\langle x_{0} x_{2} x_{4}, x_{1} x_{3} x_{5}\right\rangle \\
\left\langle x_{3}^{2} x_{0}+x_{5}^{2} x_{2}+x_{1}^{3} x_{4}, x_{4}^{2} x_{1}+x_{0}^{2} x_{3}+x_{2}^{3} x_{5}\right\rangle \\
\left\langle x_{1} x_{2} x_{3}+x_{3} x_{4} x_{5}+x_{5} x_{0} x_{1}, x_{2} x_{3} x_{4}+x_{4} x_{5} x_{0}+x_{0} x_{1} x_{2}\right\rangle .
\end{gathered}
$$


Proof. This is a straightforward computation.

Since all the 2-dimensional representations are mutually isomorphic this defines a $\mathbf{P}^{3}$ of pencils of cubics.

Proposition 7.7. For every $G_{6}$-orbit of 3 lines in $\mathbf{P}^{5}$, there is a unique pencil of $G_{6}$-invariant pencils of cubics containing these lines.

Proof. We can assume that the 3 lines in question are $\left\langle e_{0}, e_{3}\right\rangle,\left\langle e_{1}, e_{4}\right\rangle$ and $\left\langle e_{2}, e_{5}\right\rangle$. A general pencil of $G_{6}$-invariant pencils of cubics is of the form

$$
\begin{gathered}
a\left\langle x_{0}^{3}+x_{2}^{3}+x_{4}^{3}, x_{1}^{3}+x_{3}^{3}+x_{5}^{3}\right\rangle \\
+b\left\langle x_{0} x_{2} x_{4}, x_{1} x_{3} x_{5}\right\rangle \\
+c\left\langle x_{3}^{2} x_{0}+x_{5}^{2} x_{2}+x_{1}^{2} x_{4}, x_{4}^{2} x_{1}+x_{0}^{2} x_{3}+x_{2}^{2} x_{5}\right\rangle \\
+d\left\langle x_{1} x_{2} x_{3}+x_{3} x_{4} x_{5}+x_{5} x_{0} x_{1}, x_{2} x_{3} x_{4}+x_{4} x_{5} x_{0}+x_{0} x_{1} x_{2}\right\rangle .
\end{gathered}
$$

Such a pencil contains the above lines if and only if $a=c=0$.

Remark 7.8. (i) It is also easy to determine the pencil of pencils containing the other minimal $H_{6}$-orbits of lines. E.g. the three lines fixed by the elements $\sigma$ and $\tau^{3}$ are the line $\langle(1,1,1,1,1,1),(1,-1,1,-1,1,-1)\rangle$ and its $\tau$-translates. The corresponding pencil of pencils is given by $3 a+b=c+d=0$.

(ii) Every pencil of $G_{6}$-invariant pencils of cubics containing a minimal orbit $\left\{L_{1}, L_{2}, L_{3}\right\}$ also contains the three $\mathbf{P}^{3^{\prime}} s$ spanned by two of these lines. (This can be seen by direct inspection). Hence every such pencil has a base locus consisting of three $\mathbf{P}^{3^{\prime}} s$ and a residual 3 -fold $X$ of degree 6. By Proposition $6.1, X$ is an elliptic scroll. Furthermore, every elliptic 3 -fold scroll $X_{F}$, as in section 3 , is $G_{6}$-invariant, so it is contained in a $G_{6}$-invariant pencil of cubics. Hence for a general pencil of cubics the residual $X$ must be of the form $X_{E}$ for a suitable elliptic curve $E$.

Our next aim is to study the Heisenberg action on the embedded abelian surfaces $E \times F$. Recall that every abelian surface with a very ample $(1,6)$-polarization can be embedded $G_{6}$-equivariantly into $\mathbf{P}^{5}$ and that the choice of such an embedding is equivalent to the choice of a level- 6 structure on $E \times F$ (i.e. a canonical level struture associated to the polarization $H$ by which we mean a symplectic basis of the kernel of the map $\lambda_{H}: A \rightarrow \hat{A}$ ). We denote the family of all $G_{6}$-invariant abelian surfaces in $\mathbf{P}^{5}$ with two plane elliptic fibrations by $\mathcal{A}$. As in section 1 we start with curves $E$ and $F$ and a $3: 1$ morphism $\gamma: E \rightarrow F$. We can assume that $E=\mathbf{C} /(\mathbf{Z} \tau+\mathbf{Z}), F=\mathbf{C} /(\mathbf{Z} 3 \tau+\mathbf{Z})$ and that the map $\gamma$ is induced by $\gamma(z)=3 z$. We denote the generators $\tau$ and 1 of the lattice $\mathbf{Z} \tau+\mathbf{Z}$ by $e_{1}$ and $e_{3}$ and set $s_{6}=e_{1} / 6$ and $t_{6}=e_{3} / 6$. Moreover we denote the generators $3 \tau$ and 1 of the lattice $\mathbf{Z} 3 \tau+\mathbf{Z}$ by $e_{2}$ and $e_{4}$. Then $\gamma\left(e_{1}\right)=e_{2}, \gamma\left(e_{3}\right)=3 e_{4}$. The point $u_{6}=\gamma\left(s_{6}\right)$ is represented by $e_{2} / 6$ and $\gamma\left(t_{6}\right)=3 v_{6}$ is represented by $e_{4} / 2$. We choose $v_{6}$ as the point represented by $e_{4} / 6$. In this set-up the product $A=E \times F$ is given by the 
period matrix

$$
\left(\begin{array}{cccc}
\tau & 0 & 1 & 0 \\
0 & 3 \tau & 0 & 1
\end{array}\right) .
$$

The first step is to understand the polarization $H=2 E+\Gamma$ in terms of a Riemann form. This is necessary to understand the level- 6 structures on $A$. First we look at the semi-positive line bundle defined by $E$. The corresponding Riemann form with respect to the lattice $L=\mathbf{Z} e_{1}+\mathbf{Z} e_{2}+\mathbf{Z} e_{3}+\mathbf{Z} e_{4}$ is clearly given by

$$
H_{E}=\left(\begin{array}{cccc}
0 & 0 & 0 & 0 \\
0 & 0 & 0 & 1 \\
0 & 0 & 0 & 0 \\
0 & -1 & 0 & 0
\end{array}\right) .
$$

Next we want to identify the form $H_{\Gamma}$ with respect to the chosen basis $e_{1}, \ldots, e_{4}$. Since $\Gamma \cdot F=1$ we can write $A=\Gamma \times F$. By abuse of notation, let $\gamma=($ id, $\gamma)$ : $\mathrm{E} \rightarrow \mathrm{E} \times \mathrm{F}$ by the embedding of $E$ into $A$. Then $\gamma(E)=\Gamma$. We have $\gamma\left(e_{1}\right)=$ $e_{1}+e_{2}=: f_{1}, \gamma\left(e_{3}\right)=e_{3}+3 e_{4}=: f_{2}$. We can also choose $f_{1}, f_{2}, e_{2}, e_{4}$ as a basis for $L$. With respect to this basis the semi-positive form $H_{\Gamma}$ is given by $\left(e_{2}, e_{4}\right)=1$ and all other products 0 . A straightforward calculation then shows that in terms of the basis $e_{1}, \ldots, e_{4}$ the form $H_{\Gamma}$ is given by:

$$
H_{\Gamma}=\left(\begin{array}{cccc}
0 & 0 & 3 & -1 \\
0 & 0 & -3 & 1 \\
-3 & 3 & 0 & 0 \\
1 & -1 & 0 & 0
\end{array}\right) .
$$

Since $H=2 E+\Gamma$ it follows that the corresponding form with respect to the basis $e_{1}, \ldots, e_{4}$ is given by

$$
H=\left(\begin{array}{cccc}
0 & 0 & 3 & -1 \\
0 & 0 & -3 & 3 \\
-3 & 3 & 0 & 0 \\
1 & -3 & 0 & 0
\end{array}\right) .
$$

Note that this is indeed the form associated to a $(1,6)$-polarization, since

$$
\operatorname{det}\left(\begin{array}{cc}
3 & -1 \\
-3 & 3
\end{array}\right)=6 .
$$

Our next aim is to identify the group $\Theta(H)=\operatorname{ker}\left(\lambda_{H}: A \rightarrow \hat{A}\right)$ as a subgroup of $A^{(6)}=E^{(6)} \times F^{(6)}$. What we have to do is to find a basis of $L^{\vee} / L$ where $L^{\vee}$ is the dual lattice with respect to the form $H$. General theory tells us that $L^{\vee} / L \cong \mathbf{Z} / 6 \times$ $\mathbf{Z} / 6$. It is a straightforward calculation to check that $e_{1} / 2+e_{2} / 6, e_{3} / 6+e_{4} / 2 \in L$. We can take these elements as generators of $L^{\vee} / L$. Note that as points in $A=E \times F$ these are just the points $\left(3 s_{6}, u_{6}\right)$ and $\left(t_{6}, 3 v_{6}\right)$.

Let $w_{1}=e_{1} / 2+e_{2} / 6, w_{2}=e_{3} / 6+e_{4} / 2$. Then a straightforward calculation shows that for the Weil pairing with respect to $H$ we have

$$
\left(w_{1}, w_{2}\right)=\left(e^{2 \pi i / 6}\right),
$$


i.e. these points define a level- 6 structure. Note that $2 w_{1} \in F=\{0\} \times F$ and $2 w_{2} \in E=E \times\{0\}$. In particular the 2 groups of order 3 generated by $2 w_{1}$ and $2 w_{2}$ each respect one of the two plane elliptic fibrations of $A=E \times F$. Since the embedded abelian surface $A=E \times F$ is $G_{6}$-invariant the same holds for the scrolls $X_{E}$ and $X_{F}$ defined by the 2 plane cubic fibrations. Since the groups of order 3 generated by $2 w_{1}$, resp. $2 w_{2}$ each respect one of these fibrations it follows that they act trivially on the 3 singular lines of the scroll $X_{E}$, resp. $X_{F}$. In particular this gives 2 groups $H\left(K_{i}\right)$ and $H\left(K_{j}\right)$ which each has the 3 singular lines of the scrolls $X_{E}$, resp. $X_{F}$ as one of its orbits. Any other choice of a level-6 structure on $A$ gives an analogous picture. We shall return to this in a moment.

We now want to understand the variety $\mathcal{A}$ parametrizing $G_{6}$-invariant abelian surfaces with 2 plane cubic fibrations. We have already observed that each of these two fibrations determines a singular scroll $X_{E}$, resp. $X_{F}$ and a group $H\left(K_{i}\right)$, resp. $H\left(K_{j}\right)$. The abelian surface $A$ is the intersection of the scrolls $X_{E}$ and $X_{F}$ (cf. Proposition 3.5.) This defines a decomposition of the family $\mathcal{A}$ into six families $\mathcal{A}_{i j}$ where $\{i, j\} \subset\{1,2,3,4\}$. We want to exhibit a concrete parametrization of the families $\mathcal{A}_{i j}$, thereby also showing that the $\mathcal{A}_{i j}$ form six irreducible components. To do this we go back to an elliptic curve $E$ as before and the level-6 structure on $E$ given by $\left(s_{6}, t_{6}\right)$. We can perform the above construction and associate to these data the surface $A=E \times F$, the polarization $H=2 E+\Gamma$ and the level-6 structure $\left(w_{1}, w_{2}\right)$. This gives us a morphism

$$
\psi_{i j}: X^{0}(6) \rightarrow \mathcal{A}_{i j}
$$

from the (open) elliptic modular curve $X^{0}(6)$ parametrizing elliptic curves with a level-6 strucure to $\mathcal{A}_{i j}$. Note that the (compact) modular curve $X(6)$ is an elliptic curve.

Lemma 7.9. The map $\psi_{i j}: X^{0}(6) \rightarrow \mathcal{A}_{i j}$ is surjective onto the component $\mathcal{A}_{i j}$ and has degree 3 .

Proof. Here we shall treat the case where $K_{i}$ and $K_{j}$ are the groups determined by $\left\langle\sigma^{2}\right\rangle$ and $\left\langle\tau^{2}\right\rangle$. This is no loss of generality. Going back to the abelian surface $A=E \times F$ we want to study the possible embeddings of $A$ into $\mathbf{P}^{5}$ such that $A \in \mathcal{A}_{i j}$. Since $G_{6}$-invariant embeddings of $A$ correspond to the choice of a level-6 structure $\left(w_{1}^{\prime}, w_{2}^{\prime}\right)$ we have to look for those level-6 structures $\left(w_{1}^{\prime}, w_{2}^{\prime}\right)$ such that $2 w_{1}^{\prime} \in F=\{0\} \times F$ and $2 w_{2}^{\prime} \in E=E \times\{0\}$ or $2 w_{1}^{\prime} \in E$ and $2 w_{2}^{\prime} \in F$. These two cases correspond to changing the role of $E$ and $F$ and it is, therefore, enough to look at the first possibility, namely $2 w_{1}^{\prime} \in F$ and $2 w_{2}^{\prime} \in E$. Since $\left(w_{1}, w_{2}\right)$ is a basis of $\Theta(H)$ we can write $w_{1}^{\prime}=\alpha w_{1}+\beta w_{2}, w_{2}^{\prime}=\gamma w_{1}+\delta w_{2}$. Moreover $2 w_{1} \in F$ and hence $2 w_{1}^{\prime} \in F$ if and only if $2 \beta w_{2} \in F$ which is only the case for $\beta=0$ or 3. Moreover $w_{1}^{\prime}$ has to be an element of order 6 . This gives us three possibilities for $\pm w_{1}^{\prime}$, namely $w_{1}, w_{1}+3 w_{2}, 2 w_{1}+3 w_{2}$. Since $\left(w_{1}^{\prime}, w_{2}^{\prime}\right)$ and $\left(-w_{1}^{\prime},-w_{2}^{\prime}\right)$ define the same level- 6 structure we can assume that $w_{1}^{\prime}$ is one of the 3 points above. A similar argument can be applied to $w_{2}^{\prime}$ and altogether we find the following 6 
possibilities for level-6 structure $\left(w_{1}^{\prime}, w_{2}^{\prime}\right)$ with $2 w_{1}^{\prime} \in F$ and $2 w_{2}^{\prime} \in E$ :

$$
\begin{gathered}
\left(w_{1}, w_{2}\right),\left(w_{1}, w_{2}+3 w_{1}\right),\left(w_{1}+3 w_{2}, w_{2}\right), \\
\left(-2 w_{1}+3 w_{2}, w_{2}+3 w_{1}\right),\left(-2 w_{1}+3 w_{2},-2 w_{2}+3 w_{1}\right),\left(w_{1}+3 w_{2},-2 w_{2}+3 w_{1}\right) .
\end{gathered}
$$

For every pair $\left(w_{1}^{\prime}, w_{2}^{\prime}\right)$ as above the pair $\left(-w_{2}^{\prime}, w_{1}^{\prime}\right)$ is a level-6 structure with $-2 w_{2}^{\prime} \in E, 2 w_{1}^{\prime} \in F$. In this way we obtain 12 level-6 structures which belong to the pair $\{i, j\}$. This fits in with the number of level-6 structures which is given by

$$
\frac{1}{2} 6^{3}\left(1-\frac{1}{4}\right)\left(1-\frac{1}{9}\right)=72=12 \cdot 6,
$$

where 6 corresponds to the number of pairs $\{i, j\} \subset\{1,2,3,4\}$.

The map $\psi_{i j}: X^{0}(6) \rightarrow \mathcal{A}_{i j}$ was defined by associating to an elliptic curve $E$ with level-6 structure $\left(s_{6}, t_{6}\right)$ the abelian surface $A=E \times F$ with level-6 structure $\left(w_{1}, w_{2}\right)$. We want to prove that this map is surjective which implies in particular that $\mathcal{A}_{i j}$ is irreducible. So we have to show that we can obtain all abelian surfaces $A=E \times F$ and all level- 6 structures $\left(w_{1}^{\prime}, w_{2}^{\prime}\right)$ as above by varying $E$ and the level-6 structure $\left(s_{6}, t_{6}\right)$. Let $\left(s_{6}^{\prime}, t_{6}^{\prime}\right)$ be any level-6 structure on $E$. To this we associate a surface $A^{\prime}=E \times F^{\prime}$ with $F^{\prime}=E /\left\langle 2 t_{6}^{\prime}\right\rangle$. If we want that $F^{\prime}=F$ we must (at least for general $F$ ) have that $\left\langle 2 t_{6}^{\prime}\right\rangle=\left\langle 2 t_{6}\right\rangle$. Moreover $t_{6}^{\prime}$ must be a point of order 6 . Up to sign this leaves us with the possibilities $t_{6}^{\prime}=t_{6}, 3 s_{6}+t_{6}, 3 s_{6}+2 t_{6}$. Altogether we obtain 18 possible level- 6 structures, namely

$$
\begin{gathered}
\left(s_{6}+2 i t_{6}, t_{6}\right),\left(s_{6}+(2 i+1) t_{6}, t_{6}\right) \\
\left(s_{6}+2 i t_{6}, 3 s_{6}+t_{6}\right),\left(-2 s_{6}+(2 i+1) t_{6}, 3 s_{6}+t_{6}\right) \\
\left(-2 s_{6}+(2 i+1) t_{6}, 3 s_{6}-2 t_{6}\right),\left(s_{6}+(2 i+1) t_{6}, 3 s_{6}-2 t_{6}\right)
\end{gathered}
$$

where in each case $i=0,1,2$. Each of the values $i=0,1,2$ gives the same level-6 structure on $A$. Hence under the morphism $\psi_{i j}: X^{0}(6) \rightarrow \mathcal{A}_{i j}$ the pairs $\left(E,\left(s_{6}^{\prime}, t_{6}^{\prime}\right)\right)$ are mapped $3: 1$ to $\left(A=E \times F,\left(w_{1}^{\prime}, w_{2}^{\prime}\right)\right)$ where $\left(w_{1}^{\prime}, w_{2}^{\prime}\right)$ runs through all 6 possible level-6 structures on $A$ with $2 w_{1}^{\prime} \in F$ and $2 w_{2}^{\prime} \in E$. In particular the map $X^{0}(6) \rightarrow \mathcal{A}_{i j}$ is $3: 1$ and surjective and $\mathcal{A}_{i j}$ is irreducible.

Next we consider the family $\mathcal{V}$ of $G_{6}$-invariant scrolls which arise as $\mathbf{P}^{2}$-scrolls defined by a plane cubic fibration of a $G_{6}$-invariant abelian surface $A$. Such a scroll is singular along 3 lines which form an orbit of one of the groups $H\left(K_{i}\right)$ and hence there is a natural decomposition $\mathcal{V}=\mathcal{V}^{1} \cup \mathcal{V}^{2} \cup \mathcal{V}^{3} \cup \mathcal{V}^{4}$ where $\mathcal{V}^{i}$ is the set of those scrolls which are invariant under $H\left(K_{i}\right)$. These scrolls are in $1: 1$ correspondence with points in an open set of the pencil of pencils of cubics associated to the group $H\left(K_{i}\right)$ (cf. Remark 7.8. ii). In particular the varieties $\mathcal{V}^{i}$ are irreducible and rational.

The $G_{6}$-action on the elliptic scrolls restricts to pencils of $G_{6}$-invariant abelian surfaces. We describe these before we return to the surfaces which lie on two scrolls. 
Recall that the normalization $\varphi: \mathbf{P}_{F}(\mathcal{E}) \rightarrow X_{F} \subset \mathbf{P}^{5}$ of the scroll $X_{F}$ is a $\mathbf{P}^{2}$-bundle over the elliptic curve $F$ associated to the rank 3 vector bundle

$$
\mathcal{E}=\mathcal{L}_{0} \oplus h^{*} \mathcal{L}_{0} \oplus\left(h^{2}\right)^{*} \mathcal{L}_{0}, \quad\left(h^{3}=\mathrm{id}\right) .
$$

$X_{F}$ is singular along 3 lines $L_{i}$, and

$$
\left(h^{i}\right)^{*} \mathcal{L}_{0}=\varphi^{*} \mathcal{O}_{L_{i}}(1), \quad i=0,1,2
$$

are line bundles of degree 2 on $F$. If we assume that say

$$
L_{0}=\left\langle e_{0}, e_{3}\right\rangle, \quad L_{1}=\left\langle e_{1}, e_{4}\right\rangle, \quad \text { and } \quad L_{2}=\left\langle e_{2}, e_{5}\right\rangle \text {, }
$$

then

$$
\operatorname{Stab}\left(L_{i}\right)=\left\langle\tau, \sigma^{3}\right\rangle .
$$

Recall from section 3 that we may find $\mathbf{P}_{F}(\mathcal{E})$ as a quotient of $\mathbf{P}^{2} \times E$ by a subgroup $\mathbf{Z} / 3 \subset H_{3}$. This subgroup leaves three sections $E \rightarrow \mathbf{P}^{2} \times E$ invariant, and these three sections are mapped in the quotient to the three sections $F \rightarrow \mathbf{P}_{F}(\mathcal{E})$ which again are mapped $2: 1$ to the singular lines in $X_{F}$. In this set-up we may describe the $G_{6}$-action on the abelian surfaces on $X_{F}$. These surfaces are all pulled back to anticanonical divisors on $\mathbf{P}_{F}(\mathcal{E})$. Recall that $\mathrm{h}^{0}\left(\mathcal{O}_{\mathbf{P}(\mathcal{E})}(-K)\right)=4$. Elements in $|-K|$ pull back to products

$$
E^{\prime} \times E \subset \mathbf{P}^{2} \times E
$$

where $E^{\prime}$ is a cubic curve invariant under the subgroup of order 3 ; in suitable coordinates it is defined by a form in the web

$$
\left\langle x_{0}^{3}, x_{1}^{3}, x_{2}^{3}, x_{0} x_{1} x_{2}\right\rangle .
$$

These forms are precisely the invariants of degree 3 of a subgroup of order 3 in $H_{3}$. In fact the elements of order 3 of $H_{6}$ which fix the lines $L_{i}$ pointwise, leave each plane in $X_{F}$ invariant and the lift to an action on $\mathbf{P}_{F}(\mathcal{E})$ and $\mathbf{P}^{2} \times E$ which leaves each plane invariant and fixes the three special sections pointwise. These sections, in suitable coordinates, meet each plane in the points $(1,0,0),(0,1,0),(0,0,1)$ and the action is given by $\tau \in H_{3}$ in the plane.

The involution $\iota$ leaves $L_{0}$ fixed while $L_{1}$ and $L_{2}$ are interchanged. This also lifts to $\mathbf{P}^{2} \times E$. The $\iota$-invariant plane cubics in the above web form the net

$$
\left\langle x_{0}^{3}, x_{1}^{3}+x_{2}^{3}, x_{0} x_{1} x_{2}\right\rangle .
$$

$G_{6}$ finally permutes the three lines cyclically, so the corresponding plane cubics are defined by invariant forms in the variables permuted cyclically. This action is the one defined by $\sigma \in H_{3}$. The $G_{6}$-invariant anticanonical divisors therefore correspond precisely to the Hesse-pencil

$$
\left\langle x_{0}^{3}+x_{1}^{3}+x_{2}^{3}, x_{0} x_{1} x_{2}\right\rangle .
$$


As an $H_{3}$-module $\mathrm{H}^{0}\left(\mathcal{O}_{\mathbf{P}^{2}}(3)\right)$ splits as the Hesse-pencil plus 8 characters. Two of these give rise to anticanonical divisors on $\mathbf{P}_{F}(\mathcal{E})$. The remaining 6 characters of $H_{3}$ give rise to bielliptic surfaces in $|-K+T|$ where $T$ is some torsion divisor.

At this point we can also understand the different $G_{6}$-embeddings of a scroll $X_{F}$ into $\mathbf{P}^{5}$. For this we start with a $G_{6}$-invariant scroll $X_{F}$. Its desingularisation is $\mathbf{P}_{F}(\mathcal{E})$. First note that the Heisenberg group $H_{2}$ acts on the planes of $X_{F}$ and that this induces actions of $H_{2}$ on the base curve $F$ of $\mathbf{P}_{F}(\mathcal{E})$ and on the 3 sections which are mapped to the singular lines of $X_{F}$. To define a non-degenerate map from $\mathbf{P}_{F}(\mathcal{E})$ to $\mathbf{P}^{5}$ is the same as defining an isomorphism from $H^{0}\left(\mathcal{O}_{\mathbf{P}_{F}(\mathcal{E})}(1)\right)=$ $H^{0}(\mathcal{E})$ to $V$. The decomposition $\mathcal{E}=\mathcal{L}_{0} \oplus h^{*} \mathcal{L}_{0} \oplus\left(h^{2}\right)^{*} \mathcal{L}_{0}$ defines a decomposition $H^{0}(\mathcal{E})=U_{1} \oplus U_{2} \oplus U_{3}$ where each of the spaces $U_{i}$ is the space of sections of a degree 2 line bundle on $F$ and hence has dimension 2. The level 2 structure on $F$ which comes from the action of $G_{6}$ on $X_{F}$ gives us an identification (unique up to a scalar) of each of the $U_{i}$ with the $H_{2}$-module $U$. Now we pick one of the 4 subgroups $K_{j}$. Recall that as an $H_{2}$-module $V=3 U$, whereas as an $H\left(K_{j}\right)$-module $V=U \oplus U^{\prime} \oplus \bar{U}^{\prime}$ (cf. Lemma 7.3). In order to map the 3 decomposing sections of $\mathbf{P}_{F}(\mathcal{E})$ to the singular lines associated to the group $K_{j}$ we must map each of the spaces $U_{i}$ to one of the spaces $U, U^{\prime}$ and $\bar{U}^{\prime}$. The group $G_{6}$ acts transitively on the lines associated to $K_{j}$ and hence we can (up to an element in $G_{6}$ ) assume that $U_{1}, U_{2}, U_{3}$ map to $U, U^{\prime}, \bar{U}^{\prime}$. This defines the isomorphism from $H^{0}(\mathcal{E})$ to $V$ up to an element in $\left(\mathbf{C}^{*}\right)^{3}$. On the other hand the Heisenberg group $H_{3}$ acts irreducibly on the 3-dimensional space given by the decomposition $V=3 U$. Hence, by Schur's lemma, the isomorphism from $H^{0}(\mathcal{E})$ to $V$ is uniquely defined (up to a scalar). This shows that given a $G_{6}$-invariant embedding of $X_{F}$ in $\mathbf{P}^{5}$ we can find four such embeddings, one for each of the subgroups $K_{j}$.

We can now consider the incidence correspondence

$$
\mathcal{I}(A, X)=\{(A, X) \mid A \in \mathcal{A}, X \in \mathcal{V}, A \subset X\} \subset \mathcal{A} \times \mathcal{V} .
$$

Proposition 7.10. This is a $2: 3$ correspondence.

Proof. Clearly $A \in \mathcal{A}$ is the intersection of two scrolls. On the other hand, the number of such surfaces in a scroll is the answer to the question: how many abelian surfaces are there in the pencil $H \subset\left|-K_{\mathbf{P}_{F}(\mathcal{E})}\right|$ coming from the Hesse pencil which are isomorphic to the product $E \times F$ ? For this we want to find an embedding $E \subset \mathbf{P}^{2} \times E$ which after projection to $\mathbf{P}^{2}$ maps $E$ to an element in the Hesse pencil which is $\mathbf{Z} / 3$-equivariant (here $\mathbf{Z} / 3$ is the group which acts on $\mathbf{P}^{2} \times E$ with quotient $\mathbf{P}_{F}(\mathcal{E})$ ). To embed $E$ as an element in the Hesse pencil is the same as choosing a level 3 structure on $E$. Say $\mathbf{Z} / 3$ acts on $E$ by translation with an element $\sigma^{\prime}$ of order 3. So we have to ask in how may ways we can extend $\sigma^{\prime}$ to a level 3 structure. If $\tau^{\prime}$ is another 3 -torsion point with $\left(\sigma^{\prime}, \tau^{\prime}\right)=1$ (here $($, ) is the Weil pairing), we have the possibilities $\left(\sigma^{\prime}, \tau^{\prime}\right),\left(\sigma^{\prime}, \tau^{\prime} \sigma^{\prime}\right),\left(\sigma^{\prime}, \tau^{\prime} \sigma^{\prime 2}\right)$ and no others. This gives us the three possibilities. 
Remark 7.11. Notice that the three choices of $\mathbf{Z} / 3$-subgroups of $\mathbf{Z} / 3 \times \mathbf{Z} / 3$ correspond precisely to the three subgroups $K_{i}$ distinct from the subgroup $K_{j}$ which stabilizes the three singular lines of the scroll $X_{F}$.

Corollary 7.12. Given two distinct subgroups $K_{i}$ and $K_{j}$, the incidence correspondence $\mathcal{I}(A, X)$ defines a $1: 1$ correspondence between elliptic scrolls $X$ whose singular lines are invariant under these two subgroups.

Proof. A scroll singular along one of the triples of lines contains exactly one abelian surface which forms the intersection with a scroll singular along the other triple of lines.

Corollary 7.13. The abelian surfaces with two plane cubic curve fibrations are contained in precisely a pencil of $G_{6}$-invariant pencils of cubic hypersurfaces.

Proof. The space of $G_{6}$-invariant pencils of cubics is a $\mathbf{P}^{3}$, and the $G_{6}$-invariant scrolls singular along a triple of lines are defined by points on four lines in $\mathbf{P}^{3}$ corresponding to the four subgroups $K_{i}$. An abelian surface in the intersection of two scrolls is contained in the pencils of cubics corresponding to a line joining two of these lines. If there were more than a pencil of invariant pencils of cubics through the surface, then it would be contained in four scrolls. This is impossible (see Proposition 7.10).

Consider the Grassmannian of lines in the space $\mathbf{P}^{3}$ of $G_{6}$-invariant pencils of cubics. The four lines of pencils defining $G_{6}$-invariant scrolls are pairwise disjoint. The lines corresponding to abelian surfaces $A \in \mathcal{A}_{i j}$ define a one to one correspondence between two skew lines, so they form a conic section in the Grassmannian. Summing up we have 6 disjoint conic sections in the Grassmannian parametrizing $\mathcal{A}$.

We can now sum up our discussion as follows:

Proposition 7.14. The variety $\mathcal{A}$ consists of 6 irreducible components $\mathcal{A}_{i j}$ indexed by the pairs $\{i, j\} \subset\{1,2,3,4\}$. The incidence variety $\mathcal{I}(A, X)$ consists of 12 components $\mathcal{I}_{i j}^{k}$ indexed by pairs $\{i, j\}$ and an element $k \in\{i, j\}$. For every component $\mathcal{I}_{i j}^{k}$ there is a diagram

$$
\begin{gathered}
X^{0}(6) \underset{p}{\stackrel{\varphi}{\longrightarrow}} \mathcal{I}_{i j}^{k} \stackrel{q}{\longrightarrow} \mathcal{V}^{k} \\
\mathcal{A}_{i j}
\end{gathered}
$$

where $\varphi$ is $3: 1$ and $p$ and $q$ are $1: 1$.

Proof. The map $\varphi$ is the map which associates to each pair $\left(E,\left(s_{6}, t_{6}\right)\right)$ the abelian surface $A=E \times F$, the level 6 structure $\left(w_{1}, w_{2}\right)$ and the scroll $X$ which is the $\mathbf{P}^{2}$ - 
scroll attached to $A$ which is $K_{k}$-invariant. This map factors through $\mathcal{A}_{i j}$ (giving the map $\psi_{i j}$ of Lemma 7.9) and in particular the projection $p$ has an inverse.

The map $q$ is $1: 1$ by Corollary 7.12 .

Remark 7.15. The components $\mathcal{A}_{i j}, \mathcal{I}_{i j}^{k}$ and $\mathcal{V}^{k}$ are all rational. We have already observed this for the varieties $\mathcal{V}^{k}$ which are isomorphic to an open set of a pencil of cubics. Since the maps $p$ and $q$ are birational, this is also true for the other varieties.

For our conclusion on 3-folds $Y=X_{E} \cup X_{F}$ we study a $G_{6}$-invariant rational cubic scroll. It plays a crucial role when we later bilink $Y$ to a 3 -fold of degree 7 (cf. Propositions 4.7 and 5.2).

The subgroups $H\left(K_{i}\right)$ have a nontrivial intersection, namely the subgroup $\left\langle\tau^{3}, \sigma^{3}\right\rangle$. This subgroup therefore fixes all 4 triples of lines. We shall find small $G_{6}$-orbits of planes intersecting all these lines. Consider the subgroup

$$
G_{3}=\left\langle\sigma^{2}, \tau^{2}, \iota\right\rangle \subset G_{6} .
$$

We look for $G_{3}$-invariant planes.

Now the action of $\tau^{2}$ is defined by

$$
\tau^{2}=\operatorname{diag}\left(1, \eta, \eta^{2}, 1, \eta, \eta^{2}\right),
$$

where $\eta=e^{2 \pi i / 3}$ while $\sigma^{2}$ sends $x_{i} \mapsto x_{i+2}$ and $\iota$ sends $x_{i} \mapsto x_{-i}$. This implies easily that the 4 planes in the $G_{6}$-orbit of any such plane must be of the form

$$
\begin{array}{lllll}
\alpha x_{0}+\beta x_{3} & =\alpha x_{2}+\beta x_{5} & =\alpha x_{4}+\beta x_{1} & =0 & P_{0} \\
\alpha x_{0}-\beta x_{3} & =\alpha x_{2}-\beta x_{5} & =\alpha x_{4}-\beta x_{1} & =0 & P_{1} \\
\beta x_{0}+\alpha x_{3} & =\beta x_{2}+\alpha x_{5} & =\beta x_{4}+\alpha x_{1} & =0 & Q_{0} \\
\beta x_{0}-\alpha x_{3} & =\beta x_{2}-\alpha x_{5} & =\beta x_{4}-\alpha x_{1} & =0 & Q_{1}
\end{array} .
$$

In particular we notice that there is a 1-parameter family of such planes. The union of these planes forms a rational cubic scroll $R$ : In fact the union of the planes is defined by

$$
\operatorname{rank}\left(\begin{array}{ccc}
x_{0} & x_{2} & x_{4} \\
x_{3} & x_{5} & x_{1}
\end{array}\right) \leq 1,
$$

so the scroll $R$ is the Segre embedding of $\mathbf{P}^{1} \times \mathbf{P}^{2}$ in $\mathbf{P}^{5}$.

Remark 7.16. The orbits of planes in $R$ do not all have length 4 , in fact

$$
P_{0} \cap P_{1}=\emptyset, \quad Q_{0} \cap Q_{1}=\emptyset
$$

$$
\text { and } \quad P_{0} \cap Q_{0}=P_{0} \cap Q_{1}=P_{1} \cap Q_{0}=P_{1} \cap Q_{1}=\emptyset \Leftrightarrow \alpha^{2} \neq \pm \beta^{2} \text {. }
$$

Hence the orbit is 4 disjoint planes unless $\alpha^{2}= \pm \beta^{2}$. In case $\alpha^{2}=-\beta^{2}$ let $\alpha=1$. Then $\beta= \pm i$ and $P_{0}=Q_{1}, P_{1}=Q_{0}$. While in case $\alpha^{2}=\beta^{2}$, let $\alpha=1$. Then $\beta= \pm 1$ and $P_{0}=Q_{0}, P_{1}=Q_{1}$. Thus the 4 planes are disjoint unless 2 planes 
come together, which happens for

$$
(\alpha, \beta)=(1,0),(0,1),(1,1),(1,-1),(1, i),(1,-i),(1,1),(1,-1),
$$

altogether 4 pairs of planes.

Remark 7.17. The cubic scroll $R$ contains all four sets of $G_{6}$-invariant triples of lines. These lines are all transverse to the planes of $R$. Thus the six singular lines of two scrolls $X_{E}$ and $X_{F}$ which intersect along an abelian surface $E \times F$ are all contained in a rational cubic scroll.

In Lemma 8.1 we shall show that the union $Y=X_{E} \cup X_{F}$ is contained in 6 quintics. These are all singular along two triples of lines in the scroll $R$. We analyze these quintics more closely. With bihomogeneous coordinates $s, t$ and $y_{0}, y_{1}, y_{2}$ on $R \cong \mathbf{P}^{1} \times \mathbf{P}^{2}$, the restriction of quintics singular along the two triples of lines generated by $\tau$ and $\sigma$ have the form

$$
\begin{gathered}
y_{0}^{2} y_{1}^{3}-y_{0}^{3} y_{1} y_{2}-y_{0} y_{1}^{2} y_{2}^{2}+y_{0}^{2} y_{2}^{3} \\
y_{0}^{3} y_{1}^{2}-y_{0} y_{1}^{3} y_{2}-y_{0}^{2} y_{1} y_{2}^{2}+y_{1}^{2} y_{2}^{3} \\
y_{0}^{2} y_{1}^{2} y_{2}-y_{0}^{3} y_{2}^{2}-y_{1}^{3} y_{2}^{2}+y_{0} y_{1} y_{2}^{3},
\end{gathered}
$$

multiplied by any quintic in $s, t$. The 6 quintics in the ideal of two scrolls are determined by a pencil of quintics in $s, t$. This pencil is $G_{3}$-invariant, so it is an element in the net

$$
\left\langle s^{5}, t^{5}\right\rangle \oplus\left\langle s^{4} t, s t^{4}\right\rangle \oplus\left\langle s^{3} t^{2}, s^{2} t^{3}\right\rangle
$$

of pencils. The basepoints of this pencil define precisely the planes of $R$ common to all quintics through $W$. If the pencil has no basepoints, then the two scrolls would intersect $R$ in only the singular lines. We shall see in Proposition 7.18 that this is not the case. On the other hand every point of intersection of the two scrolls with $R$ outside the 6 singular lines lies in a plane which must be defined by a basepoint of the pencil, so there are at most 4 planes with such an intersection. These planes clearly form orbits under $G_{6}$ so there are 2 or 4 planes as explained above.

We carry this analysis a bit further in order to show that the union of two scrolls is bilinked to a Del Pezzo 3-fold $W_{7}$. First we consider the intersection of the cubic scroll $R$ and an elliptic scroll $X$. We use the fact that $X$ is $G_{6}$-invariant and that each plane in $R$ is invariant under the subgroup $G_{3} \subset G_{6}$ generated by $\left\langle\sigma^{2}, \tau^{2}\right\rangle$. Thus any point in a plane has an orbit by this subgroup of order divisible by 3 , and any invariant curve has degree divisible by 3 .

Proposition 7.18. The intersection $X \cap R$ is a curve of degree 18. It has the following decomposition into irreducible components:

$$
C=2 L_{1}+2 L_{2}+2 L_{3}+l_{1}+\ldots+l_{12}
$$

where $L_{i}$ are the singular lines of $X$ and as such meet every plane in $R$. The lines $l_{i}$ form 4 triangles in 4 planes of the $\mathbf{P}^{2}$-bundle $R$. 
Proof. First we prove that the intersection is a curve. If not, it contains an irreducible surface, call it $T$. If $T$ contains a plane, this is common to $R$ and $X$. But no plane in $X$ is stabilized by $G_{3}$, in fact $X$ intersects only one of the 4 triples of lines, so this is impossible. Thus $T$ intersects each plane in $R$ in some curve. This curve has degree 3 since $X$ is contained in two cubics and the curve is invariant under $G_{3}$.

As noted above, the three singular lines of the scroll $X$ all lie in $R$, and are transverse to the planes in $R$. Since every cubic through $X$ is singular along the three lines, the intersection of a cubic with $R$ is a triangle in each plane. In fact $T$ in $R=\mathbf{P}^{2} \times \mathbf{P}^{1}$ must equal $T=T_{0} \times \mathbf{P}^{1}$, where $T_{0}$ is this triangle, i.e. $T$ is the union of three quadric surfaces. But $X$ does not contain any quadric surface, so the intersection $X \cap R$ cannot contain a surface. It is therefore a curve, call it $\Gamma$. Since the intersection is proper, this curve has, by Bezout, degree 18 .

We have seen already that the curve $\Gamma$ contains the three singular lines. In fact on $\tilde{X}$, the normalization of $X$, the preimage $\tilde{\Gamma}$ of $\Gamma$ contains the curves $F_{i}$ which lie $2: 1$ over the singular lines $L_{i}$. Thus on $\tilde{X}$ we have

$$
\tilde{\Gamma}=F_{1}+F_{2}+F_{3}+a h^{2}+b h f,
$$

in notation as in the proof of Proposition 4.10 , with $6 a+b=12$. Since any plane which intersects $R$ properly intersects in a scheme of length 3 , the general plane of $X$ must intersect $R$ in three points, the points of intersection between the plane and the lines $L_{i}$. Therefore $a=0$, and $\tilde{\Gamma}=F_{1}+F_{2}+F_{3}+l_{1}+\ldots+l_{12}$ where the $l_{i}$ are lines in the planes of $\tilde{X}$.

Finally if a plane in $R$ intersects $X$ along a curve, this curve has degree divisible by three, in fact equal three, since $X$ lies in two cubics. Therefore the twelve lines $l_{i}$ form triangles in four planes of $R$.

\section{Conclusion}

We shall conclude by showing that the union of two scrolls $X_{E}$ and $X_{F}$ which intersect along an abelian surface $E \times F$ is bilinked to a Del Pezzo $W_{7}$.

Let $Y=X_{E} \cup X_{F}$.

Lemma 8.1. $\mathrm{h}^{0}\left(\mathcal{I}_{Y}(5)\right)=6$ and $Y$ lies on irreducible quintic hypersurfaces.

Proof. Consider the exact sequence

$$
0 \rightarrow \mathcal{I}_{Y}(5) \rightarrow \mathcal{I}_{X_{E}}(5) \oplus \mathcal{I}_{X_{F}}(5) \rightarrow \mathcal{I}_{E \times F}(5) \rightarrow 0 .
$$

First, the intersection $E \times F \cap \mathbf{P}^{3}$ of $E \times F$ with a general $\mathbf{P}^{3}$ is at least 5regular in the sense of of Castelnuovo-Mumford, so the same is true for $E \times F$, i.e. $\mathrm{h}^{1}\left(\mathcal{I}_{E \times F}(k)\right)=0$, when $k \geq 4$. In particular, $\mathrm{h}^{0}\left(\mathcal{I}_{E \times F}(5)\right)=102$. Similarly, the intersection $Y \cap \mathbf{P}^{2}$ of $Y$ with a general plane is 5 -regular in the sense of 
Castelnuovo-Mumford so $\mathrm{h}^{1}\left(\mathcal{I}_{Y}(k)\right)=0$ for $k \geq 4$. Furthermore $\mathrm{h}^{0}\left(\mathcal{I}_{X_{E}}(5)\right)=$ $\mathrm{h}^{0}\left(\mathcal{I}_{X_{F}}(5)\right)=54$ by Corollary 6.4. Therefore $\mathrm{h}^{0}\left(\mathcal{I}_{Y}(5)\right)=6$. If every quintic in the ideal of $Y$ is reducible, then they all have a fixed quartic hypersurface as a component. But $Y$ is $G_{6}$-invariant, and there are no $G_{6}$-invariant quartic hypersurfaces, so the lemma follows.

Now, according to Proposition 7.18 the two elliptic scrolls $X_{E}$ and $X_{F}$ intersect the rational scroll $R$ in the six singular lines and in four triangles each. Since $X_{E} \cap X_{F}$ is a surface which does not meet the six singular lines, the two sets of four triangles must lie pairwise in the same four planes of $R$. Thus the six quintics through the two scrolls $X_{E} \cup X_{F}$ contain these four planes. In particular, if $Z$ is linked $(5,5)$ to the union $X_{E} \cup X_{F}$, then $Z$ has degree 13 and contains four planes of $R$. Furthermore since each quintic intersects the planes in $R$ in curves singular in the six points of intersection with the singular lines, $Z$ will intersect each plane in one point in addition to the six singular points. The general quintic in the pencil intersects a plane in an irreducible curve, so, by Bezout, there are no quartic curves singular in the six singular points. Therefore any quartic hypersurface containing $Z$ must also contain $R$.

Now, the partial normalization $Y^{\prime}$ of $Y=X_{E} \cup X_{F}$ along the 6 singular lines is Calabi-Yau (cf. Proposition 3.5). In particular the dualizing sheaf $\omega_{Y}$, is trivial and has one global section. It follows from the next lemma that the dualizing sheaf $\omega_{Y}$ also has a section.

Let $f: Y^{\prime} \rightarrow Y$ be a finite morphism of projective schemes and denote by $\omega_{Y}$ the dualizing sheaf of $Y$. Then $f^{!} \omega_{Y}$ is a dualizing sheaf of $Y^{\prime}$ (See [Ha, Ex III, 7.2] and for the definition of $f^{!} \omega_{Y}$ see [Ha, Ex III, 6.10]). Hence we can put $\omega_{Y^{\prime}}=f^{!} \omega_{Y}$.

Lemma 8.2. If $\mathrm{H}^{0}\left(Y^{\prime}, \omega_{Y^{\prime}}\right) \neq 0$, then also $\mathrm{H}^{0}\left(Y, \omega_{Y}\right) \neq 0$.

Proof. Using [Ha, Ex III,6.10(b)] we have that

$$
\begin{aligned}
\mathrm{H}^{0}\left(Y^{\prime}, \omega_{Y^{\prime}}\right) & =\operatorname{Hom}_{\mathcal{O}_{Y^{\prime}}}\left(\mathcal{O}_{Y^{\prime}}, \omega_{Y^{\prime}}\right)=\operatorname{Hom}_{\mathcal{O}_{Y^{\prime}}}\left(\mathcal{O}_{Y^{\prime}}, f^{!} \omega_{Y}\right) \\
& =\mathrm{H}^{0}\left(\mathcal{H o m}_{\mathcal{O}_{Y^{\prime}}}\left(\mathcal{O}_{Y^{\prime}}, f^{!} \omega_{Y}\right)\right) \\
& =\mathrm{H}^{0}\left(f_{*} \mathcal{H o m}_{\mathcal{O}_{Y^{\prime}}}\left(\mathcal{O}_{Y^{\prime}}, f^{!} \omega_{Y}\right)\right) \\
& =\mathrm{H}^{0}\left(\mathcal{H o m}_{\mathcal{O}_{Y}}\left(f_{*} \mathcal{O}_{Y^{\prime}}, \omega_{Y}\right)\right) \quad([\text { Ha., Ex.III.6.10(b)]) } \\
& =\operatorname{Hom}_{\mathcal{O}_{Y}}\left(f_{*} \mathcal{O}_{Y^{\prime}}, \omega_{Y}\right) .
\end{aligned}
$$

Hence a section $s$ of $\omega_{Y^{\prime}}$ gives rise to a morphism $\varphi_{s}: f_{*} \mathcal{O}_{Y^{\prime}} \rightarrow \omega_{Y}$. Combining this with the natural morphism $\mathcal{O}_{Y} \rightarrow f_{*} \mathcal{O}_{Y^{\prime}}$ we obtain a morphism $\mathcal{O}_{Y} \rightarrow \omega_{Y}$ and hence a section of $\omega_{Y}$.

In the cohomology of the liaison exact sequence (cf. [PS])

$$
0 \rightarrow \omega_{Y} \rightarrow \mathcal{O}_{Y \cup Z}(4) \rightarrow \mathcal{O}_{Z}(4) \rightarrow 0,
$$


a section of $\omega_{Y}$ corresponds to a section of $\mathrm{h}^{0}\left(\mathcal{I}_{Z}(4)\right)$, i.e. to a quartic hypersurface containing $Z$. This quartic must contain $R$.

Let $W$ be linked to $Z$ in this quartic and a general quintic through $Z$. Then $W$ must intersect $R$ in a surface, in fact in a surface linked to 4 planes in the intersection of $R$ with a quintic hypersurface. This is clearly a surface of degree 11. Furthermore the arithmetic genus of $W$ is 1 by linkage, so $W \cup R$ has arithmetic genus 11. From the liaison exact sequences

$$
\begin{aligned}
& 0 \rightarrow \omega_{Z}(1) \rightarrow \mathcal{O}_{Y \cup Z}(5) \rightarrow \mathcal{O}_{Y}(5) \rightarrow 0, \\
& 0 \rightarrow \omega_{Z}(1) \rightarrow \mathcal{O}_{W \cup Z}(4) \rightarrow \mathcal{O}_{W}(4) \rightarrow 0
\end{aligned}
$$

we get that $\mathrm{h}^{0}\left(\mathcal{I}_{W}(4)\right)=\mathrm{h}^{0}\left(\mathcal{I}_{Y}(5)\right)-1=5$.

Clearly all the quartics through $W$ have to contain also $R$. Thus $W \cup R$ is contained in 5 quartics.

For later we need that the 5 quarties are minors of a $4 \times 5$ matrix with linear entries. If we can show this in a special case, we may conclude by semicontinuity that so is $W \cup R$ in general (cf. [Ell]).

We do this by considering, in the notation of Remark 7.8 , the points $(1,-3,0,0)$ and $(0,0,0,1)$ in the $\mathbf{P}^{3}$ of $G_{6}$-invariant pencils of cubics. They are points on two distinct lines corresponding to two distinct subgroups $H\left(K_{i}\right)$. The corresponding pencils of cubics each define an elliptic scroll (in fact a reducible scroll) residual to three $\mathbf{P}^{3}$ 's. The union of these scrolls lies on 6 quintics and is bilinked $(5,5)$ and $(5,4)$ to a 3 -fold of degree 7 which lies in 5 quartic hypersurfaces. These 5 quartics define a determinantal 3 -fold of degree 10 . These claims are easily checked with i.e. $[\mathrm{MAC}]$.

Recall from Proposition 4.10 that $W$ is a non-normal Del-Pezzo 3 -fold $W_{7}$ as soon as we have checked that no three of the singular lines lie in a $\mathbf{P}^{3}$, and that not all six lie in a rational normal quartic scroll, and finally that the common singular locus of the quartic hypersurfaces through $W$ is precisely the 6 singular lines. While the former two requirements follows easily from our analysis of the singular lines in the previous section, the latter requirement is easily checked in the above example. Therefore we may, by Proposition 5.2, bilink $W$ in complete intersections $(5,4)$ and $(5,5)$ to a non-normal Calabi-Yau 3-fold $Y_{t}$, non-normal only along 6 lines. Clearly, we may perform the bilinkage in a family, so the reducible 3 -fold $Y$ is a degeneration of $Y_{t}$. We have shown

Theorem 8.3. The reducible 3-fold $Y=X_{E} \cup X_{F}$ is a degeneration of irreducible non-normal Calabi-Yau 3-folds of degree 12 in $\mathbf{P}^{5}$. The general such 3-fold is singular precisely along 6 disjoint lines.

Remark 8.4. Computing the normalizer $N_{3}$ of $G_{3} \subset G L(6, \mathbf{C})$ and its representations, one may show that there is an $N_{3}$-invariant linear complex in the Grassmannian $G$ of lines in the space of $G_{6}$-invariant pencils of cubics. This defines a one to one correspondence between any two of the four lines defined by the subgroups $K_{i}$. Therefore it is natural to guess that this complex defines the correspondences 
$\mathcal{A}_{i j}$, in fact that it parametrizes the set of all $G_{6}$-invariant abelian surfaces. This is proved by Gross and Popescu [GP2].

\section{References}

[Ch] Chang, M.-C., Classification of Buchsbaum subvarieties of codimension 2 in projective space. J. Reine Angew. Math. 401 (1989), 101-112.

[DP] Decker, W., Popescu, S., On surfaces in $\mathbf{P}^{4}$ and 3 -folds in $\mathbf{P}^{5}$. In: Vector bundles in algebraic geometry, eds. Hitchin, N.J., Newstead, P.E., Oxbury, W.M. Cambridge University Press L.N.S. 208 (1995), 69-100.

[Ell] Ellingsrud, G., Sur le schema de Hilbert des variétés de codimension 2 dans $\mathbf{P}^{e}$ à cone de Cohen Macaulay. Ann. Scient. E.N.S. 4. Serie, 8 (1975), 423-432.

[ES] Ellingsrud, G., Strømme, S. A., The number of twisted cubic curves on the general quintic threefold. Math. Scand. 76 (1995), 5-34.

[Fu] Fulton, W., Intersection Theory. Springer Verlag, New York-Berlin-Heidelberg (1982).

[GP1] Gross, M., Popescu, S., Equations of $(1, d)$-polarized abelian surfaces. Math. Ann. 310 (1998), 333-377.

[GP2] Gross, M., Popescu, S., Calabi-Yau 3-folds and moduli of abelian surfaces, I and II. To appear.

[Ha] Hartshorne, R., Algebraic Geometry. GTM 52 Springer-Verlag, New York, 1977.

[Kre] Kresch, A., FARSTA, a computer program for quantum cohomology. Appendix to: Quantum Cohomology at the Mittag-Leffler Institute, edited by P. Aluffi, Scuola Normale Superiore, Pisa (1997).

[MAC] Bayer, D., Stillman, M., MACAULAY: A system for computation in algebraic geometry and commutative algebra, Source and object code available for Unix and Macintosh computers. Contact the authors, or download from zariski.harvard.edu via anonymous ftp.

[PS] Peskine, C., Szpiro, L., Liaison des variétés algébriques I. Invent. Math. 26 (1974), 271-302.

[Re] Reider, I., Vector bundles of rank 2 and linear systems on algebraic surfaces. Annals of Math. 127 (1988), 309-316.

[Rey] Reye, T., Ueber lineare Systeme und Gewebe von Flächen zweiten Grades. J. Reine Angew. Math. 82 (1877), 54-83.

Authors'email addresses: hulek@math.uni-hannover.de,ranestad@math.uio.no 